\title{
VELOCITY MEASUREMENTS OF LOW REYNOLDS NUMBER TUBE FLOW USING FIBER-OPTIC 'TECHNOLOGY
}

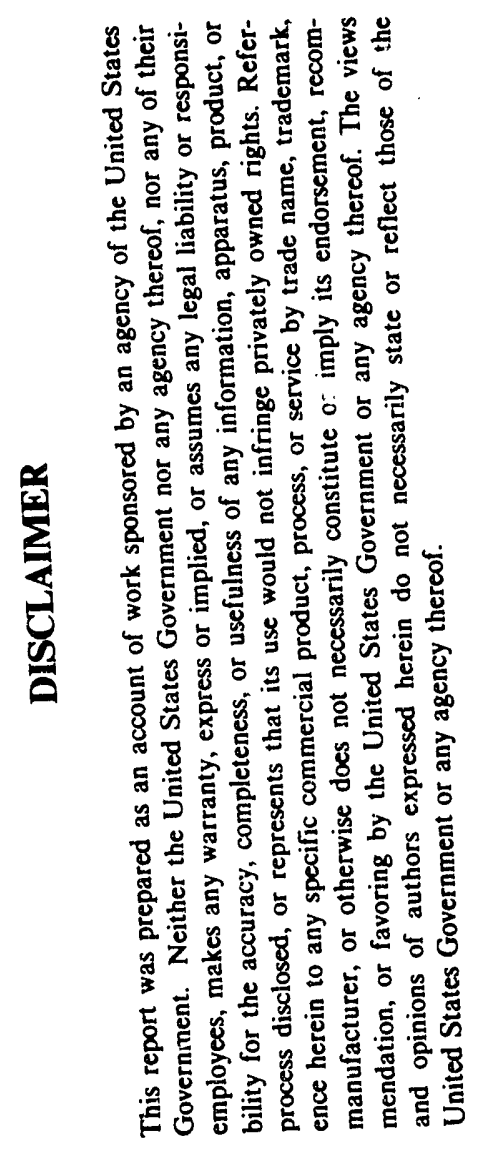

\author{
A Thesis Presented \\ by \\ J. Christopher Bianchi \\ to \\ The Faculty of the Graduate College \\ of \\ The University of Vermont \\ FGO2.91ER 6/173 \\ In Partial Fulfillment of the Requirements \\ for the Degree of Master of Science \\ Specializing in Civil Engineering
}

March, 1993 


\begin{abstract}
In 1988 Nielsen started work to measure the spatial variability of the mass flux vector of a solute that is undergoing transport in a porous medium. To measure the spatial variability of the mass flux vector, the spatial variability of its components, velocity and concentration, must be measured. Nielsen was successful in measuring the pore level concentration at many different pores and in verifying the assumption that a nonmiform concentration field exists within the mixing zone between two miscible fluids. However, Nielsen was unable to conduct the necessary pore level velocity measurements needed to measure the mass flux vector.

Nielsen's work is being continued and a probe is being developed that will measure both the velocity and concentration components at the pore level. The probe is essentially the same probe used to make the pore level concentration measurements with added capabilities needed to make the velocity measurements. This probe has several design variables, dealing primarily with the velocity component, that need further investigation before the probe may be used effectively.

The research presented in this thesis investigates these parameters by performing experiments in a capillary tube. The tube is a controlled system where the velocity of the fluid can be determined from the volumetric flow rate using Poiseuille's solution for viscous flow. Also, a statistically based relationship between the velocity measured with the probe and the velocity determined from the volumetric flow rate has been developed.
\end{abstract}




\section{Acknowledgements}

I would like to take this opportunity to thank those many people who have made this thesis possible. Most importantly, I would like to thank my advisors Dr. George Pinder and Dr. David Dongherty for allowing me the opportunity of working on this project, for their guidance and for their patience while I finished my work. I would also like to thank my committee chairperson, Dr. Jack Drake for taking a temporary leave from his sabbatical in order to serve on the committes. Also, the assistance from Berham Thran is greatly appreciated.

I also want to thank my family and friends, especially my office mates Donna and Stu, for their continued support through all of my graduate work. I want to thank my fiancée, Betsey, for serving as my editor in chief, she has read the thesis more than anyone.

I would also like to thank the IDepartment of ('ivil Engineering and the U.S. Department of Energy for the financial support that allowed me to continue my education. 


\section{Dedication}

To my fiancée, Betsey Mason, who has given me endless love and support. 


\section{Table of Contents}

Acknowledgements ................ ii

Dedication .......................... ii

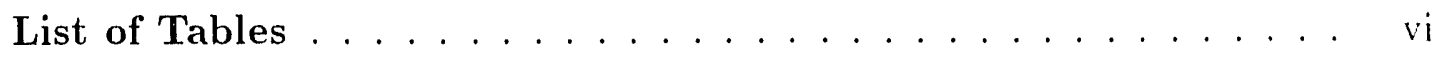

List of Figures .................... . vii

Chapter

1. Introduction . . . . . . . . . . . . . . . . 1

1.1. Research Cioals .................. 1

1.2. Earlier Work . . . . . . . . . . . . . . . . 2

1.3. Current Technology ................. 4

1.4. Background of Thesis Research ............... 6

1.5. Scope of Investigation ................ 11

2. Flow and Dispersion. . . . . . . . . . . . . 13

2.1. Review of the Volume Averaging Approach to Solnte Transport, . 13

2.2. Poisenille Flow Through a Tube . . . . . . . . . . . . 16

2.3. Dispersion in a Tube ................. 17

3. Experimental Apparatus and Materials . . . . . . . . . . 20

3.1. Design Restrictions . . . . . . . . . . . . . . 20

3.2. Data Acquisition System ................. 21

3.2.1. System (outroller................. 21

3.2.2. Pulse Transmitting System . . . . . . . . . . 22 
3.2.3. Flnorimeter System . . . . . . . . . . . . . 23

3.3. Measnement Method ................ . 26.

3.4. (apillary Tube and Flow System . . . . . . . . . . . 2s

3.5. Experimental Procedure . . . . . . . . . . . . 31

4. Discussion of Experimental Results . . . . . . . . . . . 34

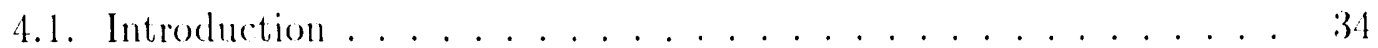

4.2. Orientation Experiment . . . . . . . . . . . 35

4.3. Radial Position of Fiber Tip . . . . . . . . . . 37

4.4. (haracteristics of the Flud Media . . . . . . . . . . . . 39)

4.5. Velocity and Pulse Duration Experiments. . . . . . . . . . 40 40

4.6. Statistical Analysis . . . . . . . . . . . . . . 42

5. Conclusions and Future Research . . . . . . . . . . . . 5)

5.1. Summary and conclusions ................ 50

5.2. Suggestions for Future Research . . . . . . . . . . . 51

Appendix

A. Programs . . . . . . . . . . . . . . . . 533

A.I. VEL.PR( . . . . . . . . . . . . . . . 53

A.2. NAWK Programs . . . . . . . . . . . . . 62

A.2.1. Normawk .................. 62

A.2.2. Fstarvawk .................. 62

B. Running the Program . . . . . . . . . . . . . . . 64

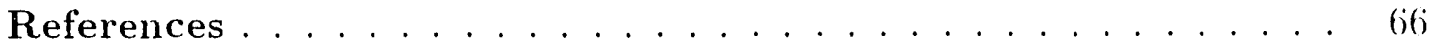




\section{List of Tables}

4.1. Maximum Volumetric Flow Rate Velocities . . . . . . . . . . . 41

4.2. SAS Ontput With Interaction T'rm . . . . . . . . . 47

4.3. SAS ()utput Without Interaction Term . . . . . . . . 48 


\section{List of Figures}

1.1. Cross-sectional view of the column to show fiber-optic placement within the column [Nies8]. Note fibers are numbered to match the legend in Figure 1.2 and do not necessarily match the numbering convention nsed in Nielsen's thesis. . . . . . . 7

1.2. Observed (symbols) and theoretical (solid curve) breakthrough curves. Each symbol corresponds to one observation point. The observation plane is at $x=78.1 \mathrm{~cm}$ and the flow rate is $5 \mathrm{ml} / \mathrm{min}$

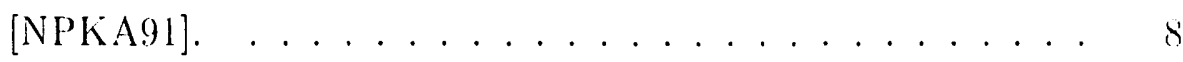

1.3. The velocity probe proposed by Behram Turan. . . . . . . . 10

2.1. The velocity distribution in a tube. . . . . . . . . . . 16

3.1. The lata acquisition system. . . . . . . . . . . 22

3.2. The pulse transmitting system circuit. . . . . . . . . . 23

3.3. The remote fiber fluorimeter $[\mathrm{LPS6}] \ldots \ldots \ldots 24$

3.4. The illumination region for a bare fiber end [HDM84]. . . . . . 25

3.5. Change in fluorimeter response due to a change in $\mathrm{pH}$ for a constant dye concentration of $15 p$ pm. . . . . . . . . . 27

3.6. a) (apillary tube dimensions, b) top view capillary tube and holder, and c) side view capillary tube and holder. . . . . . . . . 30

3.7. Flow system for capillary tube experiment. . . . . . . . . 31 
4.1. Two investigated orientations of the optical fiber relative to the pulse wire. (a) Parallel orientation and (b) perpendicular

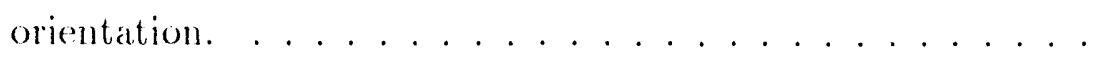

4.2. (a) Side view, (b)front view, and (c)perspective view of slug in the shape of a planar parabola in the tube. . . . . . . . 36

4.3. The re ised velocity probe used in future porous media experiments by Tiran. . . . . . . . . . . . . .

4.t. Effect of sample distance from filer tip. Each curve is the average of three repetitions at that distance. The solute concentration was $10 \mathrm{ppm}$ and the pulse duration was 1.0 see. The RFF response was normalized by subtracting the background readings from the measurements. . . . . . . . . . . .

4.5. Change in fluorimeter response due to a change in pH for a constant dye concentration of $15 p$ pm. . . . . . . . . .

4.6. Example of response curve produced from the experimental data of a run with Flow Designation (C and P'ulse Duration 1.50) scconds. The volumetric flow rate velocity, from Table 4.1, is $0.197 \mathrm{~mm} / \mathrm{sec}$, compared to $0.199 \mathrm{~mm} / \mathrm{sec}$ calculated from the First Arrival Time of 15.07 seconds. . . . . . . . . 42

4.7. Relationship for all six pulse durations. From top to bottom $P=$ 0.25 sec to $2.00 \mathrm{sec} \ldots \ldots \ldots \ldots \ldots$

4.8. Result for a pulse duration of 0.25 scconds. . . . . . . . . 44

4.9. Result for a pulse duration of 0.50 seconds. . . . . . . . . 14

4.10. Result, for a pulse duration of 0.75 seconds. . . . . . . . . . 45 
4.11. Result for a pulse duration of 1.00seconds. . . . . . . . 45

4.12. Result for a pulse duration of 1.50 seconds. . . . . . . . . . 46

4.13. Result for a pulse duration of 2.00seconds. . . . . . . . . . 46 


\section{Chapter 1 Introduction}

\subsection{Research Goals}

The research presented in this thesis is a portion of an ongoing project. A central objective of the project is to measure the spatial variability of the mass flux vector of a solute that is undergoing transport in a porous medium. To measure the spatial variability of the mass flux vector, the spatial variability of its components, velocity and concentration, must be measured. This research attempts to measure these quantities at the pore scale (microscopic scale) using fiber-optic technology.

In his master's thesis, Nielsen (1988) was able to measure the spatial variability of the concentration field as a front traveled through a column packed with glass beads.

A similar set of investigations of the variability of the velocity is currently being pursued. Behram Turan [personal communication, 1992] is attempting to use the same packed bead column used in Nielsen's work, but incorporates a probe that will measure the velocity of a tracer in addition to the concentration.

The goal of the research presented in this thesis is to develop and perform controlled experiments that will be used to test the measurement method proposed by Turan. In order to accomplish this täs, Turan's method will be applied to flow in a capillary tube. The velocity of the fluid in the tube can be calculated from the volumetric flow rate using Poisenille's solution for viscous flow. This 
research attempts to develop a relationship between the velocity calculated from the volumetric flow rate and the velocity inferred from the experimental results obtained using the proposed method.

\subsection{Earlier Work}

In the past, varions methorls have been used to measure the velocity of a fluid traveling in a tube. (iriffiths (1911) conducted a study measuring the mean speed of a fluid moving slowly through a capillary tube. In his experiments, a tube that is initially filled with slowly flowing water is fed a weak fluorescein solution. At some later time, the supply of fuorescein is stopped and replaced by water. This creates a column of color, known as an index, that slowly moves through the tube. When the column of color is at a relatively long distance from the ends of the tube, Griffiths showed that the index's center moved with the mean speed of the water. This method, although applicable to a capillary tube of small diameter, is not practical when trying to develop a method to be employed in porous media measurements for the obvious reason that the area of measurement is cleep inside the media and, therefore, it can not be viewed to make the necessary measurements.

In a similar method, a conducting fluid (brine) is injected into a tube with fluid already flowing through it. Downstrean of the injection point the conductivity of the fluid is measured continuously. As the conducting fluid passes this measuring point, the data will produce a conductivity-time curve, from which a stream velocity can be determined. This method was nsed by White (1947) to measure cardiac output. The technique used by White was used earlier by Stewart (1894), although Stewart's technique did not permit continuous measurements of the conductivity. The principles of Stewart's method were successfully applied 
by Hamilton (1928, 1932), using a non-diffusible dye instead of salt and collecting many arterial blood samples with approximately a one second interval between the samples. Hamilton's measurements produced accurate results, but his measurement technique was not widely employed because of the obvious inconvenience of collecting so many samples [Whi47].

For large water mains where fluid flow may be turbulent, Allen \& White (1923) developed the salt velocity method for measuring the discharge inside pipes (see reference [Tay53]). This method also employs the principles described above for measuring the velocity. Once the velocity and the area of the pipe are known, then the volume of water passing throngh the pipe can be determined.

Taylor (1953) developed a colorometric method for studying dispersion in a capillary tube. In his method, a strongly colored dissolved sub tance was released into a capillary tube to displace the distilled water already contained in the tube. The solution was potassium permanganate ( $1 \%$ by weight) and $99 \%$ slightly acidulated water. A comparison tube of known concentration and of identical dimensions to the experimental tube was used to determine the concentration as a function of $x$ in the experiment tube. The measurements were made by sliding the comparison tube along the side of the experiment tube until the colors in the two tubes matched. At the match point, the concentration in the experiment tube was identical to the known concentration in the comparison tube. The concentration in the comparison tube would then be changed and more comparisons would be made until the concentration field in the experiment tube was sufficiently described. In one case, Taylor released the solution as a continnous source into the tube and, when the front approached the exit, the flow in the tube was stopped. He found that the molecular diffusion was small enough that no appreciable change in color at any point in the tube could be detected 
for several hours after the flow had been stopped. Essontially, he had a still picture of the dispersion phenomenon in the tube. Furthermore, because the time from beginning to end of the solution flow was recorded, a velocity could also be determined. In another case, the potassium permanganate solution was released into the tube for only a short period and then was replaced by distilled water, thus creating a spike (or instantaneous) type source case. The concentration was determined as a function of $x$ using the same procedure described above.

The same indicator fluid concept was used by Lane and Sirs $(1973,1974)$ to measure velocity of different fluids in a straight tube undergoing laminar and well mixed flow. The indicator fluid was created by radioactively labeling solutions which were suddenly introduced into the flow stream. The labeled solutions were monitored using a NaI scintillation detector as they passed the observation point.

\subsection{Current Technology}

More recently, with the invention of fiber-optics and laser anemometry, an entirely new realm of velocimetry has been introduced. Lately, these velocimeters have been applied to measuring turbulent flow fields using an $X$ hot-wire anemometer (Brumn et al. 1990 and Acrivlellis 1989), and to measuring vector fields of a turbulent boundary layer also using hot-wire anemometry (Vukoslavcevic et al. 1991). Hot-wire anemometry is an invasive type of measurement requiring positioning of a probe at the measurement location. A very small single sensor hot-wire probe has a wire length of about $0.3 \mathrm{~mm}$. The nine-sensor hot-wire $X$ probe, used by Vukoslavcevic, has a sensing area of $1.7 \mathrm{~mm}$ vertically and $2.2 \mathrm{~mm}$ horizontally.

Some techniques which measure fluid velocity non-invasively include doppler velocimetry (Mason et al. 1979 and Durrani 1977), bidirectional time-of-flight 
velocimetry (Mason et al. 1979 and Munk et al. 1982), and second-order crosscorrelation velocimetry (Mason et al. 1979 and Beck et al. 1985). The doppler velocimetry technique uses the frequency shift associated with the reflection of waves from scatterers suspended within the flow to measure the velocity of the fluid. The bidirectional time-of-flight method uses the travel time of sound waves, propagating through the fluid, to measure the fluid's mean velocity. The secondorder cross-correlation velocimeter uses cross-correlation analysis to estimate the time delay between two line detectors which are separated by a finite distance. A further description of these methods is beyond the scope of this thesis and the interested reader is referred to the references mentioned. Further more, these methods have been successfully applied to higher flow velocities and larger diameter pipes $(v \approx 0.5 \mathrm{~m} / \mathrm{s}$ and $\phi>10 \mathrm{~mm}$. [JS91]) than those used in this research.

The principles employed in this research are essentially the same as those described by the conducting or indicator fluid method previonsly mentioned. The only difference is that instead of injecting a tracer, one is created in-situ at a known distance from the measuring point and a known location in the planar cross section of the tube. This method allows travel time to be used as a means of measuring velocity rather than having to rely on the concentration curve produced by the measurements as the means of calculating the fluid velocity. To clarify these remarks, the works of Nielsen (1988 and Nielsen et al. 1991) and Turan [personal communication, 1992] are discussed next. 


\subsection{Background of Thesis Research}

The foundation for this thesis was established by Nielsen in 1988. Nielsen examined solute transport in a uniform homogeneous porous medium with a onedimensional flow system. With fiber-optic technology, Nielsen studied the spatial variability in the pore-averaged concentration field from pore to pore within the porous medium and related the observations to the macroscopic flow characteristics. His experiments were performed in order to test the validity of the assumption that a nonuniform concentration field exists within the mixing zone between two miscible fluids. The fiber-optic technology used in Nielsen's work was first employed by Kulp et al. (1987) to monitor species concentration at several points in a packed column. Nielsen's work differed from that of Kulp et al. in that Nielsen evaluated his results in terms of dispersion [NPKA91].

The experimental apparatus was a column approximately one meter in length with an inside diameter of approximately $10 \mathrm{~cm}$. The porous media was uniformly packed 3 m.m diameter glass beads. The apparatus was designed to measure the change in the pore-averaged concentration of a fluorescent dye dissolved in a flowing fluid at several pore locations. The length and diameter of the column were designed such that apparatus-induced dispersion and wall effects would not be factors in the experiments [NPKA91].

Each sensor probe consisted of one ideal pore created from eight $3 \mathrm{~mm}$ diameter glass beads arranged in a cubic formation. The probe can be visualized by looking at the bottom eight beads in Figure 1.3. The tip of the fiber sensor was placed in this pore and the entire probe was placed in the column. Eight probes were arranged in a cross-sectional plane (see Figure 1.1) perpendicular to the direction of the flow. The fiber tips were located at two different distances from the column wall in an alternating patte $n$, four at $1.9 \mathrm{~cm}$ and the other four at $3.8 \mathrm{~cm}$. [Nies 


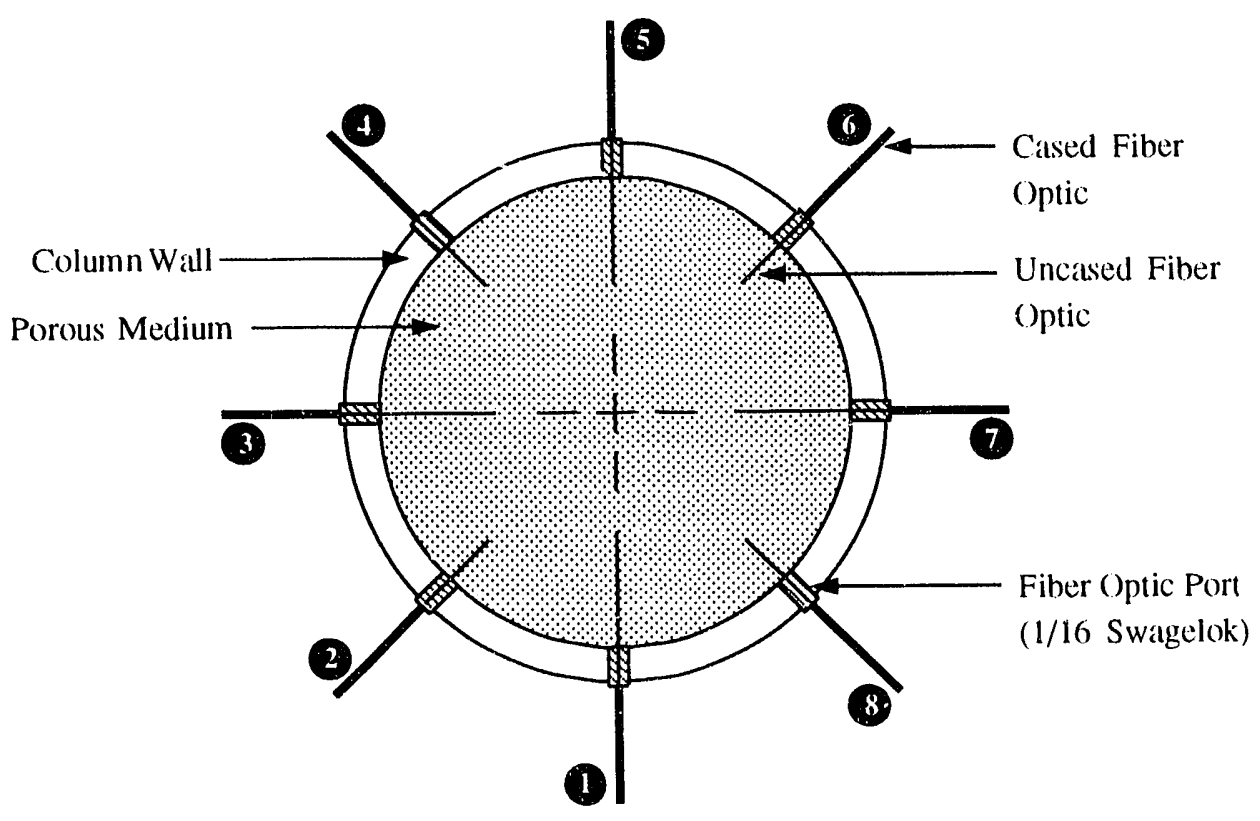

Figure 1.1: ('ross-sectional view of the column to show fiber-optic placement within the column [Nie88]. Note fibers are numbered to match the legend in Figure 1.2 and do not necessarily match the numbering convention used in Nielsen's thesis.

The tracer dye used was a solution of fluorescein sodium salt dissolved in water. The measuring system consisted of a light source, an optical fiber, a fluorimeter and an antomated data collection system. This system is similar to the one used in the experiments presented in this thesis, and thus is explained thoroughly in Section 3.2. A breakthrough curve of the displacing tracer solution was measured at each probe location. The breakthrongh curve phenomenon was caused by an increase in the concentration of the displacing tracer, thus causing an increase in the fluorescence of the solution. The fluorescence of the solution was measured by a dedicated remote fiber fluorimeter system (see Figure 3.3) [NPKA91].

Three factors were evaluated for their influence on the amount of dispersion observed in the column. The three factors were macroscopic fluid velocity, the distance from the entry point to the observation point, and the "relative time". 


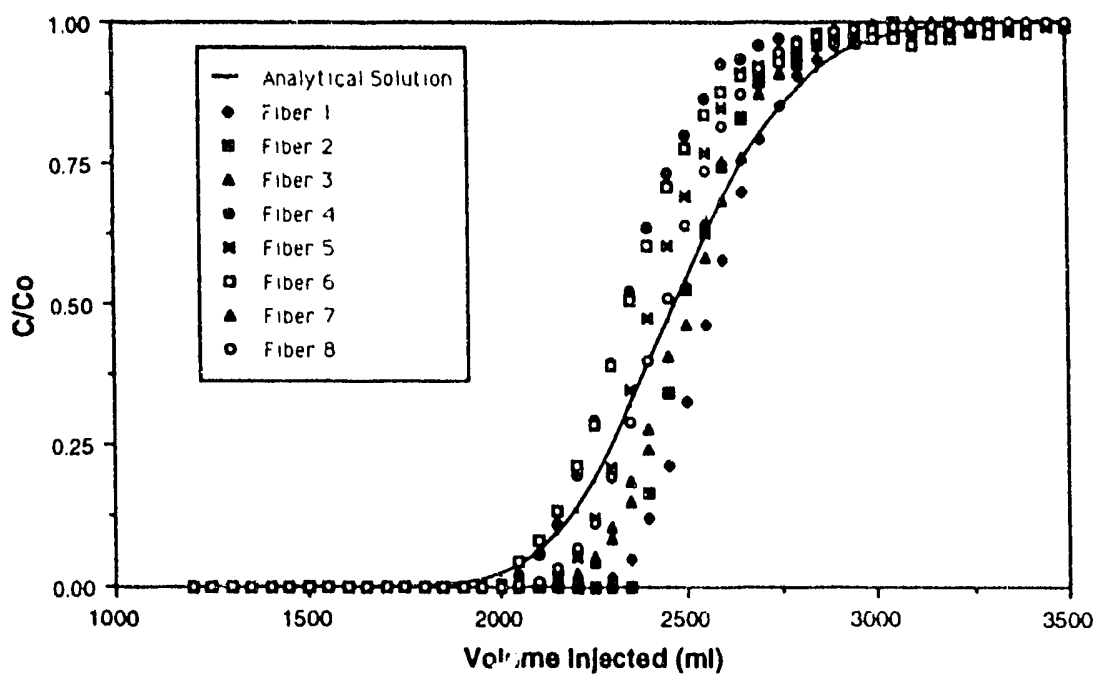

Figure 1.2: Observed (symbols) and theoretical (solid curve) breakthrough curves. Each symbol corresponds to one observation point. The observation plane is at $x=78.1 \mathrm{~cm}$ and the flow rate is $5 \mathrm{ml} / \mathrm{min}$ [NPKA91].

Three velocities, two measurement locations, and three relative times were considered. The relative time was defined as the time for the relative concentration to reach 25,50 , and $75 \%$ of the maximum. This type of experimental design is known as a $3 \times 2 \times 3$ factorial design.

From his results, Nielsen found that at some pore locations the pore-averaged concentration begins to increase earlier than at other pore locations. Nielsen presents this result in a relative concentration versus time curve (see Figure 1.2) for all the fibers at an observation plane of $78.1 \mathrm{~cm}$ from the entry point of the column. The time is presented in the form of volume injected into the column. It is important to note that the shape of the curves are consistent in Nielsen's results and that the differences in concentration are die primarily to a shift in the curve. Nielsen's results clearly show the variation in the concentration field. 
Nielsen also determined the dependence of the concentration variability on the flow rate, time, and travel distance using an analysis of variance (ANOVA) of the results from the factorial design. He defined the spatial variability in two ways. One, called $S P$, is defined as

$$
S P \equiv \frac{1}{n-1} \sum_{i=1}^{n}\left(C_{i}-\bar{C}\right)^{2} \quad \text { for } n=8
$$

where ( $\mathrm{I}$ is the porous medium average of the pore-averaged concentration taken over the porous media cross section [NPKA91]. The other definition is $M X$ and is given as

$$
M X=\text { maximum of }\left(C_{i}-C_{j}\right) \quad i, j \in I,
$$

where $I$ is the number of pore-level concentration observations in a section of porous medium [NPKA91]. Equation 1.1 defines the spatial variance in the measured pore-averaged concentration field at a specific point in time. From the ANOVA using both SSP and $M X$ definitions of the variance, Nielsen found that at the higher flow rate $(30 \mathrm{ml} / \mathrm{min})$, there was a significant increase in spatial variance at the location furthest $(73.1 \mathrm{~cm}$ ) from the inlet of the column. Whereas at the lower flow rates (5 and $15 \mathrm{ml} / \mathrm{min}$ ), there was a significant decrease in the spatial variance as the distance from the column inlet increased. He also found that the spatial variance is smaller in a range of flow rates from 15 to $25 \mathrm{ml} / \mathrm{min}$. than at lower and higher flow rates [NPKA9l].

Nielsen's work verified the assumption that a nonuniform concentration field exists within the mixing zone between two miscible fluids by measuring the spatial variation in the pore-averaged concentration field. However, in order to measure. the mass flux vector, both velocity and concentration measurements must be measured at the pore-level. Turan is continuing Nielsen's work by attempting to measure the spatial variation in pore-averaged velocity measurements within the 
porous medium, as well as performing the concentration measurements made in Nielsen's work. As Nielsen did, Turan will also relate these observations to the macroscopic flow characteristics [perconal communication, 1992].

Turan's work uses the same experimental apparatus used in Nielsen's work with modifications made to the measurement probes enabling them to make velocity measurements as well as concentration measurements. New equipment has been added to allow more measurements to be taken in less time and some modifications to the column have been made to simplify the construction process.

The probes constructed by Turan, shown in Figure 1.3, are made from the same $3 \mathrm{~mm}$ diameter glass beads that make up the porous media in the column. A probe is comprised of twelve beads cemented together in a cubic packing formation to form two adjacent ideal pores. The probe is oriented vertically in the column with

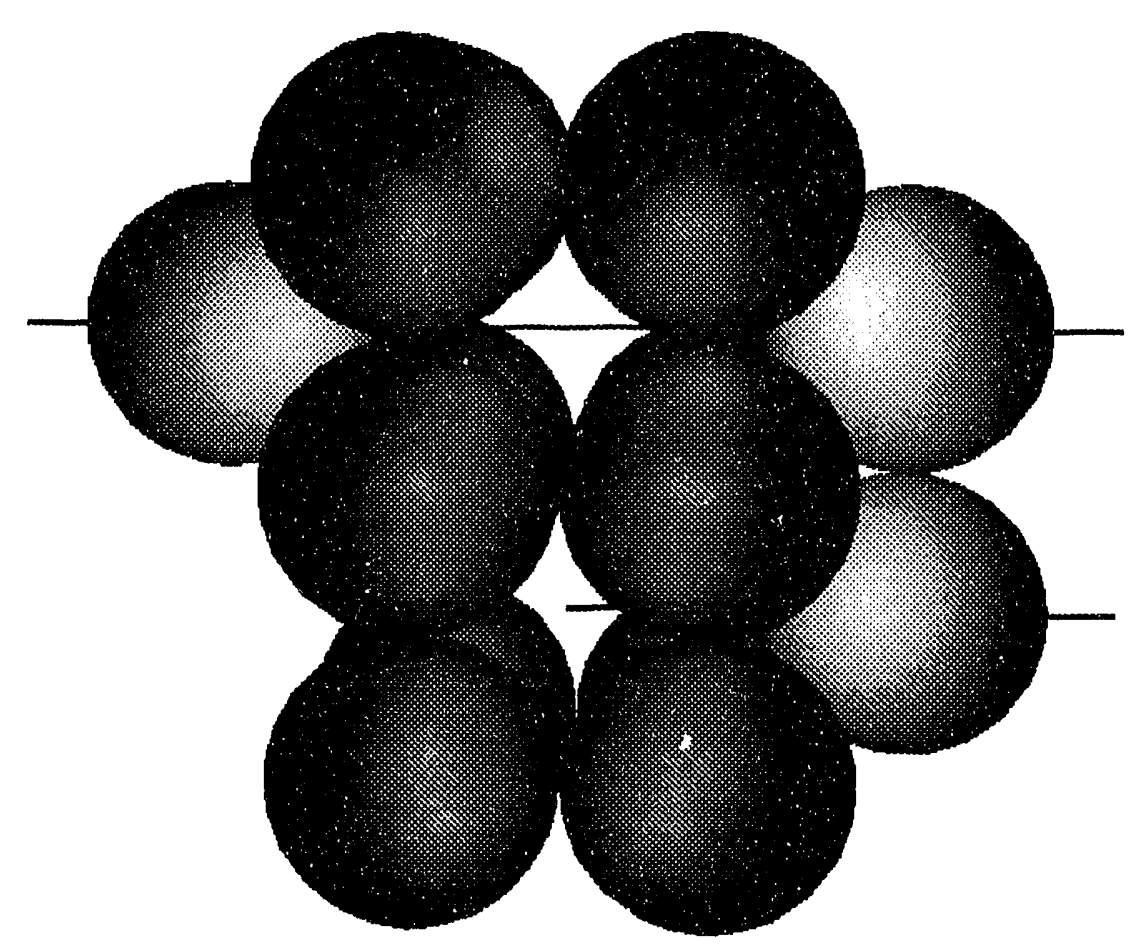

Figure 1.3: The velocity probe proposed by Behram Turan. 
the top pore containing a "pulse" wire and the bottom pore containing the fiber optrode. The pulse wire and the ontrode are held in place by $3 \mathrm{~mm}$ diameter teflon beads. A hole is drilled through the centers of these beads to guide and hold the wire and fiber. The fiber optrode and eight glass beads, comprising the bottom pore, was the original probe used to make the concentration measurements in Nielsen's work.

Figure 1.3 depicts the probe that was first proposed by Turan, which is slightly different than the probe that was finally arrived at as a result of the research presented in this thesis.

By impressing a voltage between the pulse wire in the probe and a wire mesh at the bottom of the column for a short interval of time, a change in the pH of the tracer in a region immediately surrounding the pulse wire is created. This "slug" of tracer with an altered pH travels with the fluid. When sampled by the optrode in the adjacent downstream pore, the output appears as a breakthrough curve of time and response. From the response-time curve, it is hoped that a pore scale velocity can be determined.

\subsection{Scope of Investigation}

The research presented in this thesis is motivated by the need for a better understanding of the complex probe proposed by Turan. Velocity measurements taken with the probe were found to be dependent not only on the velocity of the furd, but also on other parameters.

The velocity measurement method described in Section 1.4 has a number of design variables that have not previously been investigated. Among these are the location of the tip of the optical fiber relative to the center of a pore, the orientation of the fiber relative to that of the pulse wire, the duration of the 
pulse, and the characteristics of the Huid medium used in the experiments.

A study has been performed in order to determine the best location and orientation of the fiber optrode in relation to the centerline of the tube and the pulse wire. A comparison of the response-time curve velocities has been performed to determine what point on the curve should be used to determine the velocity. By performing a series of experiments at different volumetric flow rate velocities and comparing these values with velocities determined from the response-time curve, a relationship between the response-time curve and volumetric flow rate velocity has been developed. Also, these experiments looked at the effect of pulse duration on this relationship and found it to be a significant parameter. 


\section{Chapter 2}

\section{Flow and Dispersion}

\subsection{Review of the Volume Averaging Approach to Solute Transport}

In order to better understand the motivation for the research presented in this thesis and the motivation for the research performed by Nielsen (1988), a review of the volume averaging approach to solute transport is appropriate. Although the terms pore, microscopic, and macroscopic were introduced earlier, they will be defined here with more detail. Also, the concept of a Representative Elementary Volume, or REV, will be introduced.

Within a porous medium, such as a soil matrix or an experimental columm packed with small spherical glass beads, the fluid and solute travel through a network of interconnected voids in the medium. These interconnected voids are known as pore spaces in the medium. The pore spaces contain a liquid phase or a gaseous phase and the rest of the medium is a solid phase. The microscopic scale refers to the scale of a single pore space. The macroscopic scale encompasses many pores and describes the scale at which average properties of the porons medium are defined.

The macroscopic scale defines the domain within which we may assume there exists a representative elementary volume (REV). The spatial averaying theory, which serves as the foundation for this research, is based on the assumption that 
the REV exists. The assumption states that a macroscopic variable has a value at a point that can be obtained by averaging the microscopic values of that variable over a volume of the porous media centered at that point. The size of the REV, also known as the characteristic length, is dependent on the nature of the pores. The REV has to be large enough to represent the variability in the microscopic values and small enough so that the large scale heterogeneities of the medium do not affect the averaged values [H(i79)]

The remainder of this section discusses the equation development of the macroscopic transport equation. Because this is discussed in great detail in Nielsen's thesis (see reference [Nie88]) which follows the averaging procedure developed by Hassanizadeh and (iray (see reference [H(i79]), it will only be highlighted here.

The microscopic balance equation for solute transport within a single pore is written as

$$
\frac{\partial c}{\partial t}+\nabla \cdot(\mathbf{v} \cdot)-\nabla \cdot \mathbf{J}=0
$$

where $c$ is the solute concentration within a pore, $t$ is time, $\mathrm{v}$ is the flow velocity in a pore, and $\mathbf{J}$ represents the nonconvective flux of the solute. Every term in this equation is a microscopic quantity. Using the averaging operators defined by Hassanizadeh and Gray [H(779], each of the quantities in equation (2.1) can be transformed to represent an average of local microscopic values of that quantity. The average is taken over an REV around a point at which the value is assigned. The steps involved in the averaging procedure are not shown here (see reference [Nie88]), but the resulting equation is the macroscopic transport equation and can be expressed as

$$
\frac{\partial \bar{c}^{\alpha}}{\partial t}+\nabla \cdot\left(\overline{\mathbf{v}}^{\alpha \alpha} \bar{c}^{\alpha}\right)+\nabla \cdot\left(\mathbf{v}^{\prime \alpha} c^{\prime \alpha}\right)-\nabla \cdot\left[D_{d}\left(\nabla \bar{c}^{\alpha \alpha}+\tau\right)\right]=0 .
$$

The $\overline{\mathbf{v}}^{\mathrm{ar}}$ and $\bar{c}^{\mathrm{r}}$ terms represent the average velocity and average concentration over the alpha (fluid) phase. $D_{d}$ is the molecular diffusion constant of the solute 
and $\tau$ is a tortuousity vector which accounts for the complex flow paths in a porous medium. The third term in equation (2.2) contains the microscopic perturbation terms which are the variations from the average value of these microscopic quantities. Wntil now, these terms could not be evaluated so this term was combined with the fourth term to give the classic dispersive flux term, $\nabla \cdot(\mathbf{D} \cdot \nabla C)$. Redefining the macroscopic average terms $\overrightarrow{\mathbf{v}}^{\alpha}$ and $\bar{c}^{\alpha}$ as $\mathbf{V}$ and $C$ and replacing the third and fourth term with the dispersive Hux term, equation (2.2) becomes

$$
\frac{\partial C}{\partial t}=\nabla \cdot(\mathbf{D} \cdot \nabla C)-\mathbf{V} \cdot \nabla C
$$

where $C$ is th mencentration of solute in solution, $\mathbf{D}$ is the solute dispersion tensor (including both molecular diffusion and mechanical dispersion), and $\mathbf{V}$ is the average fluid velocity. Because Niclsen had the ability to evaluate the microscopic terms, he combined the third and fourth term in equation (2.2) by defining a single dispersive flux vector, $D^{\star}$, as

$$
\mathbf{D}^{\star}=\left(\mathbf{v}^{\prime \alpha} e^{\prime \alpha}\right)-D_{d}\left[\left(\nabla \bar{c}^{\alpha}+\tau\right)\right] .
$$

Using this new dispersive flux term, equation (2.2) can be written as

$$
\frac{\partial C}{\partial t}=\nabla \cdot\left(\mathbf{V}()^{\prime}\right)+\nabla \cdot \mathbf{D}^{\star}
$$

Because the new dispersive flux term is defined by the microscopic perturbation quantities, this macroscopic transport equation takes into account the variations in the microscopic quantities. Once the terms $\mathrm{v}^{\prime \alpha}$ and $e^{\prime \alpha}$ are measured, the two models can be compared using a numerical solution to the transport equation.

As stated earlier, Nielsen (1988) measured the term $c^{\prime c x}$ in his research work. Turan's work [personal communication 1992] and the work presented hercin are aimed at measuring the term $\mathbf{v}^{\prime \alpha}$, in addition to the $c^{\prime}$ term. 


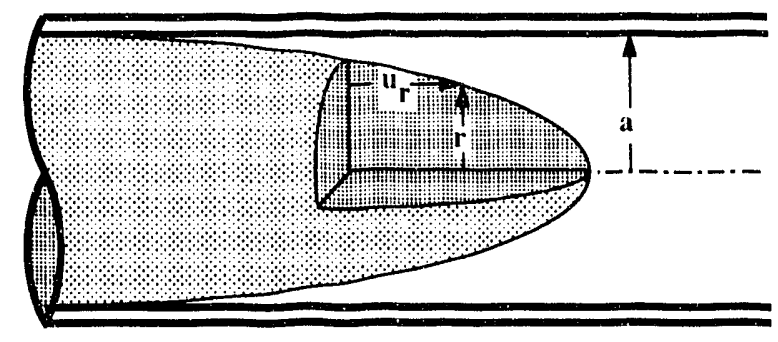

Figure 2.1: The velocity distribution in a tube.

\subsection{Poiseuille Flow Through a Tube}

Let us consider the Poisenille [SW 65 ] problem of steady laminar flow of an incompressible viscous fluid in a long capillary tube. The tube has a circular cross section with inner radius a. Let $r$ denote the radial distance from the center of the tube to a point of interest. The velocity of the fluid $u$, in the direction of the tube's long axis, is a function of raclial position,

$$
u(r)=-\frac{\left(u^{2}-r^{2}\right)}{4 \mu} \frac{d}{d l}(p+\gamma z)
$$

where $\mu$ is the viscosity of the fluid, $l$ is the length along the tube, $p$ is the pressure, $\gamma$ is the specific weight, and $z$ is the fluid elevation head. At the center of the tube, $r=0$ and $u$ is equal to the maximum velocity, $u_{m u x}$. To find the average axial velocity $\left(u_{a u g}\right)$ across the tube's axis, $u$ is integrated over the cross sectional area and dividing by the area of the tube, the resulting equation is

$$
u_{a v q g}=-\frac{a^{2}}{8 \mu} \frac{d}{d l}(p+\gamma z)
$$

From these two equations, one can see that the average velocity is one half the maximum velocity.

Because the quantity $\frac{d}{d l}(p+\gamma z)$ is constant and the integral of $u$ over the cross-sectional area is the volumetric discharge, $Q$, the derivative term in (2.6) 
can be eliminated to obtain

$$
u(r)=\frac{2 Q}{\pi a^{2}}\left[1-\left(\frac{r}{a}\right)^{2}\right] .
$$

When $\frac{r}{a}=0$, equation (2.8) reduces to the maximum velocity which is $2 Q / A$, and when $\frac{r}{n}=\frac{1}{\sqrt{2}}$ the equation reduces to the average velocity, $Q / A$.

With these definitions, we can define the average velocity $\left(u_{\text {nvg }}\right)$ in the tube as $\overline{\mathbf{u}}$, and the deviation velocity

$$
\begin{aligned}
& \mathbf{u}^{\prime}=u(r)-\overline{\mathbf{u}} \\
& \mathbf{u}^{\prime}=\left[1-2\left(\frac{r}{a}\right)^{2}\right]
\end{aligned}
$$

as is needed in dispersion theory.

\subsection{Dispersion in a Tube}

The volume averaging approach to solute transport in a porous medium, discussed in Section 2.1, can also be applied to transport in a tube (Tompson and Gray, 2. 1986). An REV can be chosen in the tulie to be a thin cross-section of width $\Delta \zeta$ in the axial $(z)$ direction. The volume of this REV is given by $\pi a^{2} \Delta \zeta$. The typical length of she averaging volume in the radial direction is equivalent to the scale of tube. Therefore, it is only possible to account for variations of the macroscopic properties in the axial direction.

Using the averaging theorems (that were used to derive the general, threedimensional macroscop c ecuations (Tompson and Gray, 1. 1986)), the general solute mass and dispersion balance equations for the tube can be given by: Mass

$$
\rho \frac{\partial \bar{\omega}}{\partial t}+\rho \frac{\partial}{\partial t}(\overline{\mathbf{u}} \bar{\omega})-\rho \mathcal{D} \frac{\partial^{2} \bar{\omega}}{\partial z^{2}}+\rho \frac{\partial f}{\partial z}-\frac{\partial \tau_{z}}{\partial z}=0
$$

Dis persion

$$
\rho \frac{\partial f}{\partial t}+\rho \overline{\mathbf{u}} \frac{\partial f}{\partial z}+\rho f \frac{\partial \overline{\mathbf{u}}}{\partial z}+\rho q_{z z} \frac{\partial \bar{\omega}}{\partial z}+\rho \frac{\partial w_{z z}}{\partial z}-\rho \mathcal{D} s_{z}+\overline{\omega^{\prime} \frac{\partial p^{\prime}}{\partial z}}-\overline{\mu \omega^{\prime} \frac{\partial^{2} \mathbf{u}^{\prime}}{\partial z^{2}}}=0
$$


In these equations, $\bar{\omega}$ and $\omega^{\prime}$ are the mass fraction $\left(\rho_{i} / \rho\right)$ average and deviation values respectively. $f$ (from Tompson and Gray, 1. 1986) is defined as $\overline{\mathrm{u}^{\prime} \omega^{\prime}}$. $\mathcal{D}$ is the constant molecular diffusivity. The tortuosity $\tau_{z}$ is a line integral and is equal to zero. The scalar quantities $q_{z z}, w_{z z}$, and $s_{z}$ are all components of the respective tensorial source terms defined (in Tompson and Cray, 1. 1986) as

$$
\begin{gathered}
q_{z z}=\overline{\mathbf{u}^{\prime} \mathbf{u}^{\prime}} \\
w_{z z}=\overline{\mathbf{u}^{\prime} \mathbf{u}^{\prime} \omega^{\prime}}, \\
s_{z}=\mathbf{u}^{\prime} \frac{\partial^{2} \omega^{\prime}}{\partial z^{2}} .
\end{gathered}
$$

The perturbation velocity term was defined in the previous section. Therefore, $q_{z z}$ can be evaluated as

$$
\begin{aligned}
q_{z z} & =\frac{1}{\pi a^{2}} \int_{0}^{a} u_{m a x}{ }^{2}\left(\frac{1}{2}-\frac{r^{2}}{a^{2}}\right) 2 \pi r d r \\
& =\frac{u_{\max }{ }^{2}}{12}=\frac{\overline{\mathbf{u}}^{2}}{3} .
\end{aligned}
$$

Because the pressure is constant across the cross section of the tube (for a laminar, parabolic flow profile), the perturbation term, $p^{\prime}$, is equal to zero for all $z$. Since $\mathbf{u}^{\prime}$ is independent of $z$, equations $(2.10)$ and $(2.11)$ can be rewritten as:

$$
\begin{gathered}
\rho \frac{\partial \bar{\omega}}{\partial t}+\rho \overline{\mathbf{u}} \frac{\partial \bar{\omega}}{\partial z}-\rho \mathcal{D} \frac{\partial^{2} \bar{\omega}}{\partial z^{2}}+\rho \frac{\partial f}{\partial z}=0 \\
\rho \frac{\partial f}{\partial t}+\rho \overline{\mathbf{u}} \frac{\partial f}{\partial z}+\rho \frac{\overline{\mathbf{u}}^{2}}{3} \frac{\partial \bar{\omega}}{\partial z}+\rho \frac{\partial w_{z z}}{\partial z}-\rho \mathcal{D} s_{z}=0
\end{gathered}
$$

In order to write equation (2.14) and (2.15) in a dimensionless form, the following dimensionless quantities need to be defined:

$$
\begin{aligned}
& \bar{V}=\overline{\mathbf{u}} / \overline{\mathbf{u}} \quad \bar{C}=\omega / \omega_{0} \quad Z=z / a \quad T=t \overline{\mathbf{u}} / a \\
& F=\overline{V^{\prime} C^{\prime}}=f / \overline{\mathbf{u}} \omega_{0} \quad S_{z}^{\prime}=\frac{s_{z} a^{2}}{\overline{\mathbf{u}} \omega_{0}} \quad W_{z z}=\frac{w_{z z}}{\overline{\mathbf{u}}^{2} \omega_{0}}
\end{aligned}
$$


where $\omega_{0}$ is $\omega$ at the center of the tube. With these quantities, equations (2.14) and (2.15) may now be written as

$$
\begin{gathered}
\frac{\partial \bar{C}}{\partial T}+\bar{V} \frac{\partial \bar{C}}{\partial Z}-\frac{1}{P_{e} e} \frac{\partial^{2} \bar{C}}{\partial Z^{2}}+\frac{\partial F}{\partial Z}=0, \\
\frac{\partial F}{\partial T}+\bar{V} \frac{\partial F}{\partial Z}+\frac{\bar{V}^{2}}{3} \frac{\partial \bar{C}}{\partial Z}+\frac{\partial W_{z z}}{\partial Z}-\frac{1}{P_{e}} S_{z}=0,
\end{gathered}
$$

where the Peclet number, Pee, is equal to $\overline{\mathbf{u}} a / \mathcal{D}$. This model is valid for pipe flow systems where $\rho, \mu$, and $\mathcal{D}$ are constant, the velocity distribution is given by equation (2.8), and there is no interphase mass transfer or chemical reactions (Tompson and Gray, 2. 1986). 


\section{Chapter 3}

\section{Experimental Apparatus and Materials}

\subsection{Design Restrictions}

As indicated earlier, the research experiments discussed in this thesis are a part of a broader project which is discussed in Section 1.4. In order to ensure that this research produces valuable information to bring the whole project closer to its goal, these experiments had to complement those experiments already performed in the earlier parts of the research. This criteria imposed certain restrictions on the design and implementation of these experiments.

It has been calculated that the $3 \mathrm{~mm}$ glass beads used in the norous media probes would yield a pore diameter of approximately $2.2 \mathrm{~mm}$. This value is arrived at by determining the largest diameter sphere that can fit inside the pore created by eight beads in a cubic packing formation. The distance between the pulse wire and the optrode in these probes is $3 \mathrm{~mm}$, which is the distance between the centers of two adjacent pores created by a cubic packing formation. These dimensions were used as an approximate lower limit for the diameter of the capillary tube. This lower limit was increased when a question arose about the effective path length of the optical fibers. The question that arose was whether or not the sampling region in front of the optrode tip is interfered with by the walls of the pores. The effective path length $\left(L_{E}\right)$, which is approximately $2 m m$, is discussed in more detail in Section 3.2.3. Another concern was how the measured response 
is affected by the location of the sample within the sampling region. Motivated by these questions, the diameter arrived at for the capillary tube was approximately $4 m m$. This diameter allows experiments to be performed that would test the optrode when the tip is positioned at the center line of the tube and the tube wall is outside the sampling region governed by the effective path length. The tube finally chosen was determined by what was readily available in the clear lucite plastic. There were two possible tubes, one with an inside diameter (I.I).) of $\frac{1}{16} \mathrm{in}$ $(1.53 \mathrm{~mm})$, which was too small, and another with an I.D) of $\frac{3}{16} \mathrm{in}(4.76 \mathrm{~mm})$.

\subsection{Data Acquisition System}

The system had three main components; a system controller, a pulse transmitting system and a fluorimeter system. Interaction between these components is illustrated in Figure 3.1, and is discussed below. The system controller was a personal computer running DOS (Disk Operating System). The pulse transmitting system had two components, a voltage source and a digital scanner. The fluorimeter system was made up of a fluorimeter, a fiberoptic multiplexer, and a digital multimeter.

\subsubsection{System Controller}

The computer used for these experiments was an AT\&T model 6300 operating under MS-DOS 3.1. The system controller was interfaced with the voltage source, the digital scanner, the multiplexer and the multimeter via an IEEE-488 communication bus. To accomplish the interface, a CPIB (General Purpose Information Bus) interface ard (Metrabyte MB(:-488) was installed in the computer.

The Lab ASYST software (MacMillan Software (o.) was used to provide a 


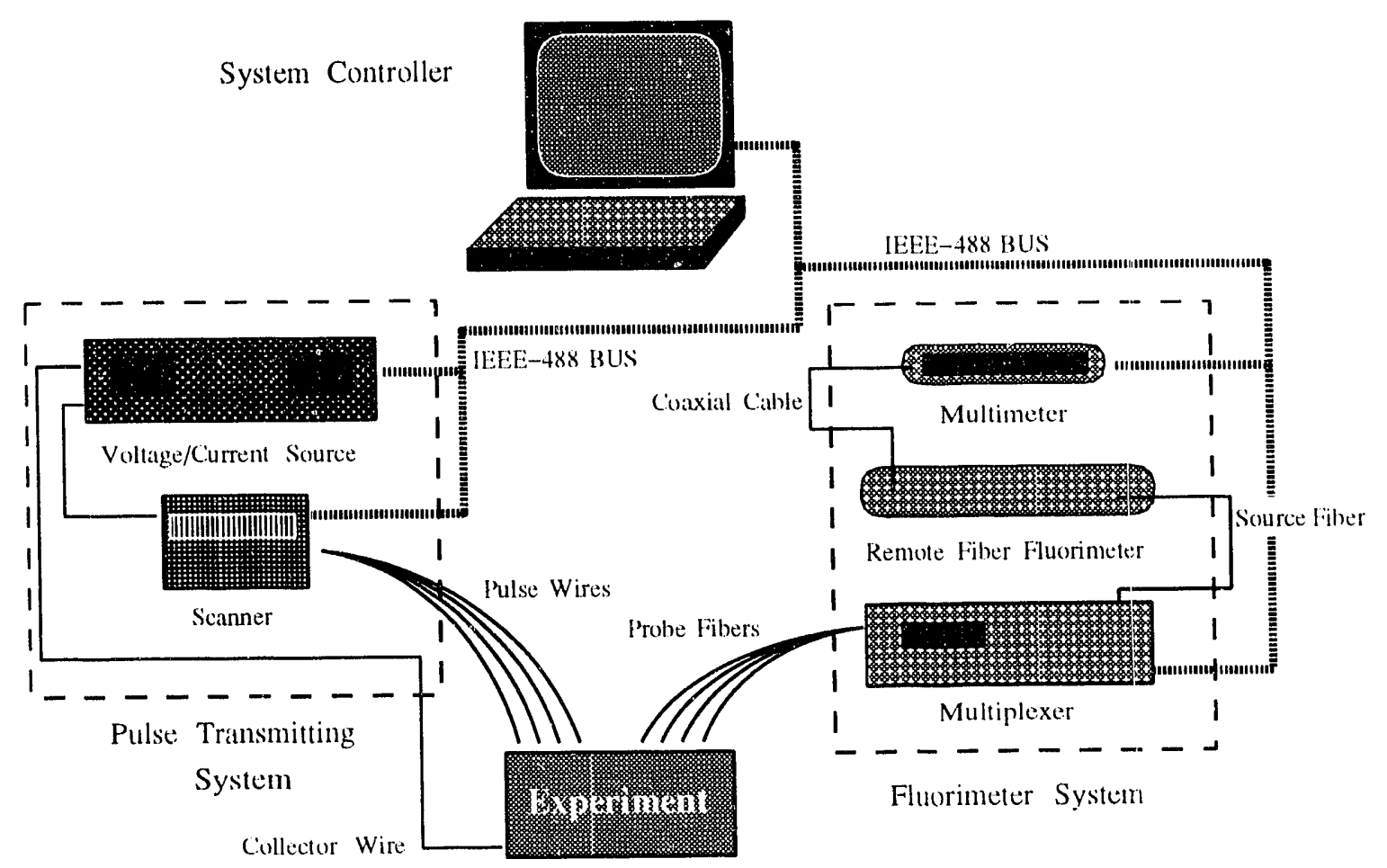

Figure 3.1: The data acquisition system.

high level programming environment. This environment enabled programs written in the AsYST language to utilize the communication link provided by the IEEE-488 bus, thus allowing antomated control of the components interfaced with the computer.

\subsubsection{Pulse Transmitting System}

The pulse transmitting system uses a Kiethley 223A Voltage/Current Source, and a Kiethley 705 scanner. The voltage/current source can be programmed to limit either the voltage or the current in the circuit. For example, by specifying a high current and a low voltage the voltage is limited. The actual current and voltage values are dependent on the resistance in the circuit. The scanner controls the duration of the pulse, and acts as a switch to control which probe receives the 
pulse. It can handle up to forty pulse wires, although these experiments use only one. The others are being used for the corresponding part of the research mentioned earlier in Section 1.4.

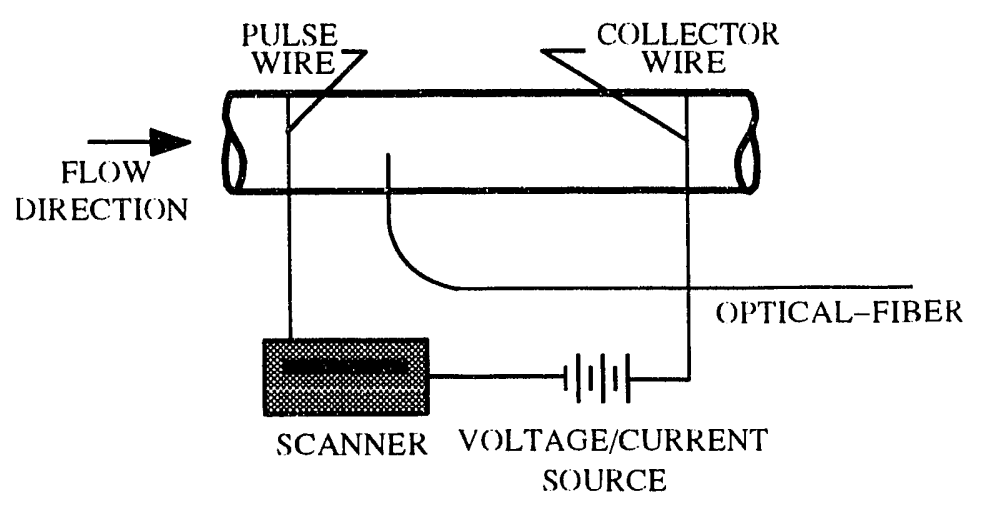

Figure 3.2: The pulse transmitting system circuit.

A diagram of the pulse circuit is stown in Figure 3.2. The system controller initializes the voltage/current source to the desired voltage or current, and also the scanner to the starting channel (pulse wire) and duration time. When signaled by the program, the scanner completes the circuit for the desired pulse duration and a voltage is applied across the pulse (source) wire and the collector (drain) wire in the tube (or probe).

\subsubsection{Fluorimeter System}

The fluorimeter system uses a Kiethley 175 Autoranging Multimeter, a DICON Multichannel Fiberoptic Switch (multiplexer), and a remote fiber fluorimeter (RFF). The RFF, shown in Figure 3.3, was designed and built by the Environmental Science Division of the Lawrence Livermore National Laboratory (LLNL), University of California. The design requires the use of only one fiber for each sensor probe which enables the probes to be smaller and reduces the interference to the fluid flow within the pore or capillary tube [Nie88]. 


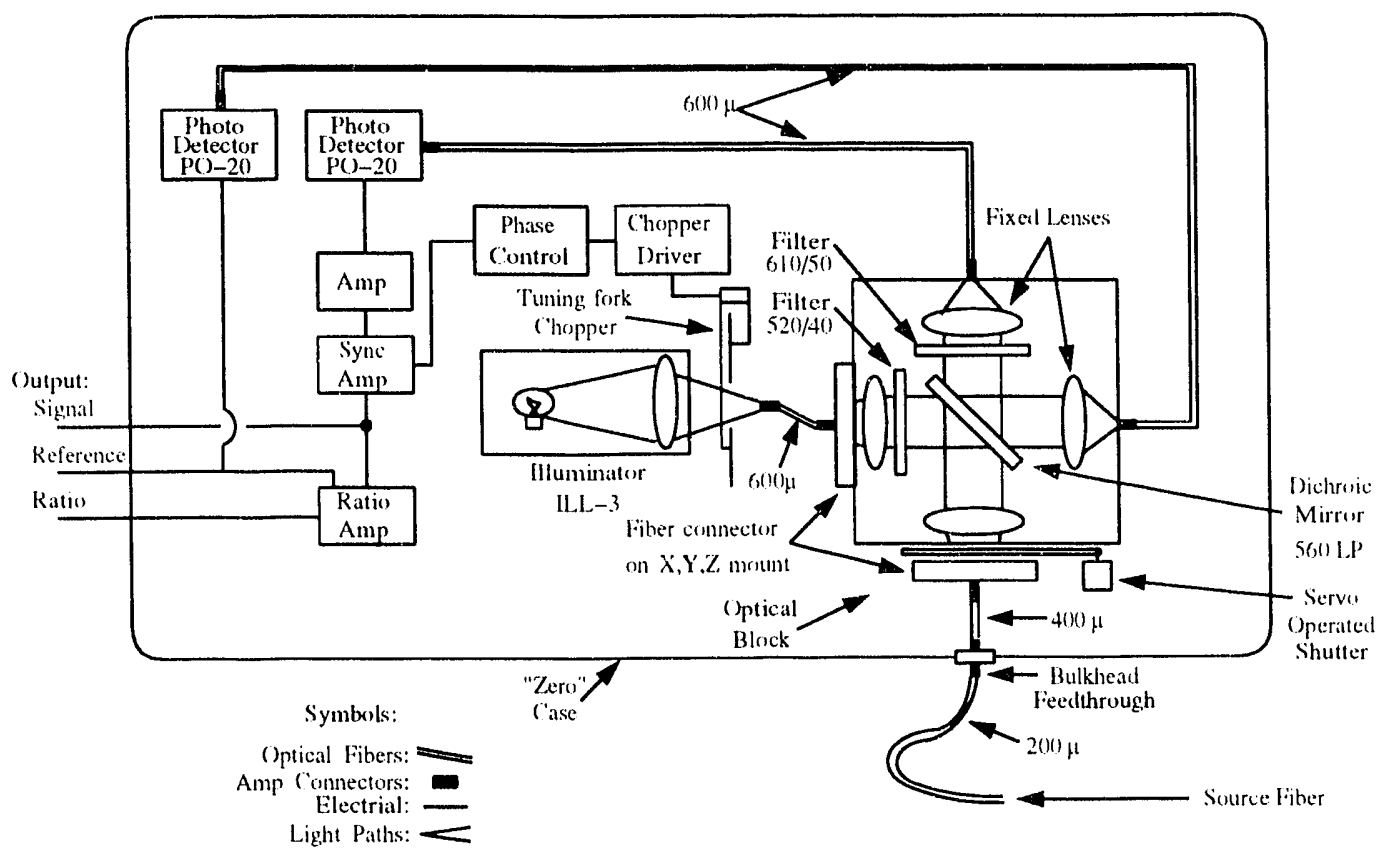

Figure 3.3: The remote fiber Huorimeter [LPS6].

The light is carried by the Source Fiber (labeled in Fignre 3.3) from the fluorimeter to the 36 channel multiplexer. The multiplexer enables the use of multiple probes with a single fluorimeter. The ASYST program communicates to the multiplexer which probe is to be sampled, and the multiplexer makes the connection between the source fiber and the fiber connected to the appropriate probe. The light reflected by the sample is carried by the this fiber back throngh the multiplexer and the source fiber to the photo detectors (see Figure 3.3) in the RFF. For the experiments in this thesis, the use of a multiplexer with 36 channels is not required, but it enables two parts of the research to be performed simultaneously. The other part, mentioned in Section 1.4 , requires 33 of the 36 channels.

Output from the fluorimeter is interpreted as a Do(: voltage source by a Kiethley $175 \mathrm{~A}$ multimeter which is comnected to the fluorimeter via a coaxial cable (see Figure 3.1). The computer receives measurements directly from the multimeter via the IEEE-488 bus. 


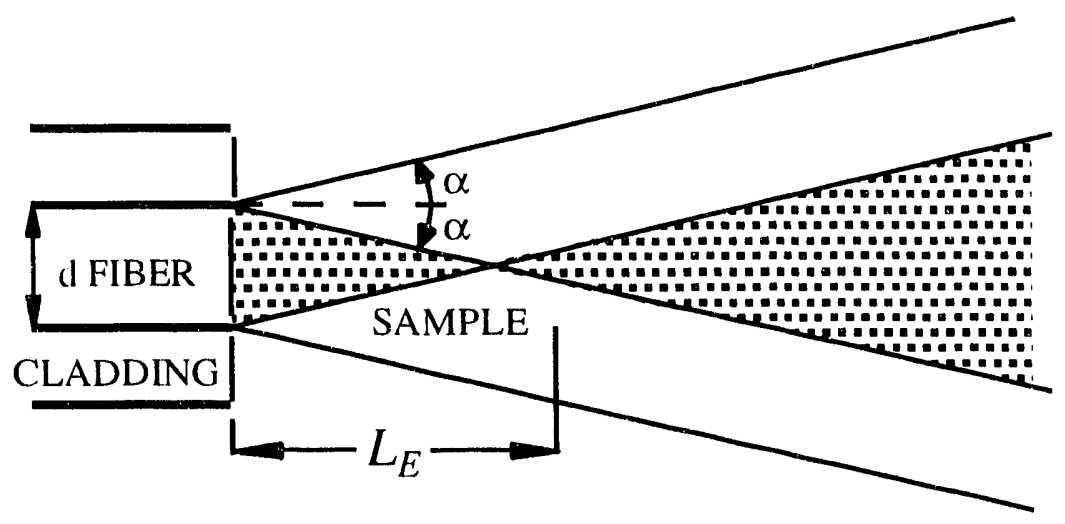

Figure 3.4: The illumination region for a bare fiber end [HDMs4].

For a more detailed description of the inner workings of the fluorimeter, the reader is referred to the masters thesis of Mark Nielson (1988, [pp 51-54]).

The fibers used in these experiments are 200/250 microns in diameter fibers with nylon jackets. The source fiber is connected to the multiplexer and the fluorimeter by metal terminators that are installed at both ends of the fiber. The sensor probe fibers are connected to the multiplexer with a metal terminator and are bare-polished at the probe end. The field of view for a bare-polished fiber is shown in Figure 3.4. The apex angle $\alpha$ defines the illumination region and is given by [HDM84]

$$
\alpha=\sin ^{-1}\left(\frac{N A}{\eta}\right),
$$

where $N A$ is the numerical aperture of the fiber and $\eta$ is the refractive index of the sample. The effective path length $L_{E}$ is given by [HDM84]

$$
L_{E}=\frac{0.727 d}{\tan \alpha}
$$

where $d$ is the fiber diameter. $L_{E}$ defines a region in front of the fiber tip in which a sample passing through this region will be effectively measured. For the fibers used in these experiments $L_{E}$ is approximately $2 \mathrm{~mm}$ [Nie88].

The fluorescent chemical used in the tracer dye was Uranine $\left(\mathrm{C}_{20} \mathrm{H}_{10} \mathrm{Na}_{2} \mathrm{O}_{5}\right.$, 
F.W. 376.28, Fisher Scientific), which is a purified grade of fluorescein sodium salt. The tracer dye is made from a stock solntion of Uranine and distilled water $(1000 \mathrm{pmm})$ and is diluted with a solution of sodium sulfate $\left(\mathrm{Na}_{2} \mathrm{~s}^{\prime} \mathrm{O}_{4}, 200 \mathrm{pmm}\right)$ and distilled water. The sodium sulfate is used to increase the conductivity of the distilled water and to maintain a consistent conductivity for each experiment. A Uranine concentration of $10 \mathrm{ppm}$ was used as the tracer dye for the experiments.

\subsection{Measurement Method}

The development of the measurement technique was derived from a number of papers (Baker, 1966; (iold, 1956; Noller, 1957; Jordan et al., 1987) which are discussed in the proposal [Pin91] to continue the research. The technique that was used in the end requires a bare-polished optical fiber and two electrodes. One electrode is placed upstream of the optrode (the bare-polished optical fiber) at some distance. This electrode is referred to as the pulse wire. The pulse wire is a 10 micron silver coated platinum core wire made using the Wollaston process (Sigmund ('ohn ('orp.). In order to make the wire even smaller and have a better contact with the fluid the silver coating is stripped from the length of wire exposed in the probe. The silver coating is stripped by placing the wire in a nitric acid bath for approximately five minutes with a $5 \mathrm{dc}$-volt charge on the wire. For these experiments, the pulse wire is at a distance of $3 \mathrm{~mm}$ upstream from the optrode. The other electrode, referred to as the collector wire, is placed downstream of the optrode. In the case of the capillary tube experiments, this electrode is a steel wire. In the case of the packed column experiments performed by Turan, the electrode is a wire mesh.

The fluorescence of the tracer dye is partly dependent on the $\mathrm{pH}$ of the solution. Figure 3.5 demonstrates that, for a constant concentration of tracer dye, the RFF 


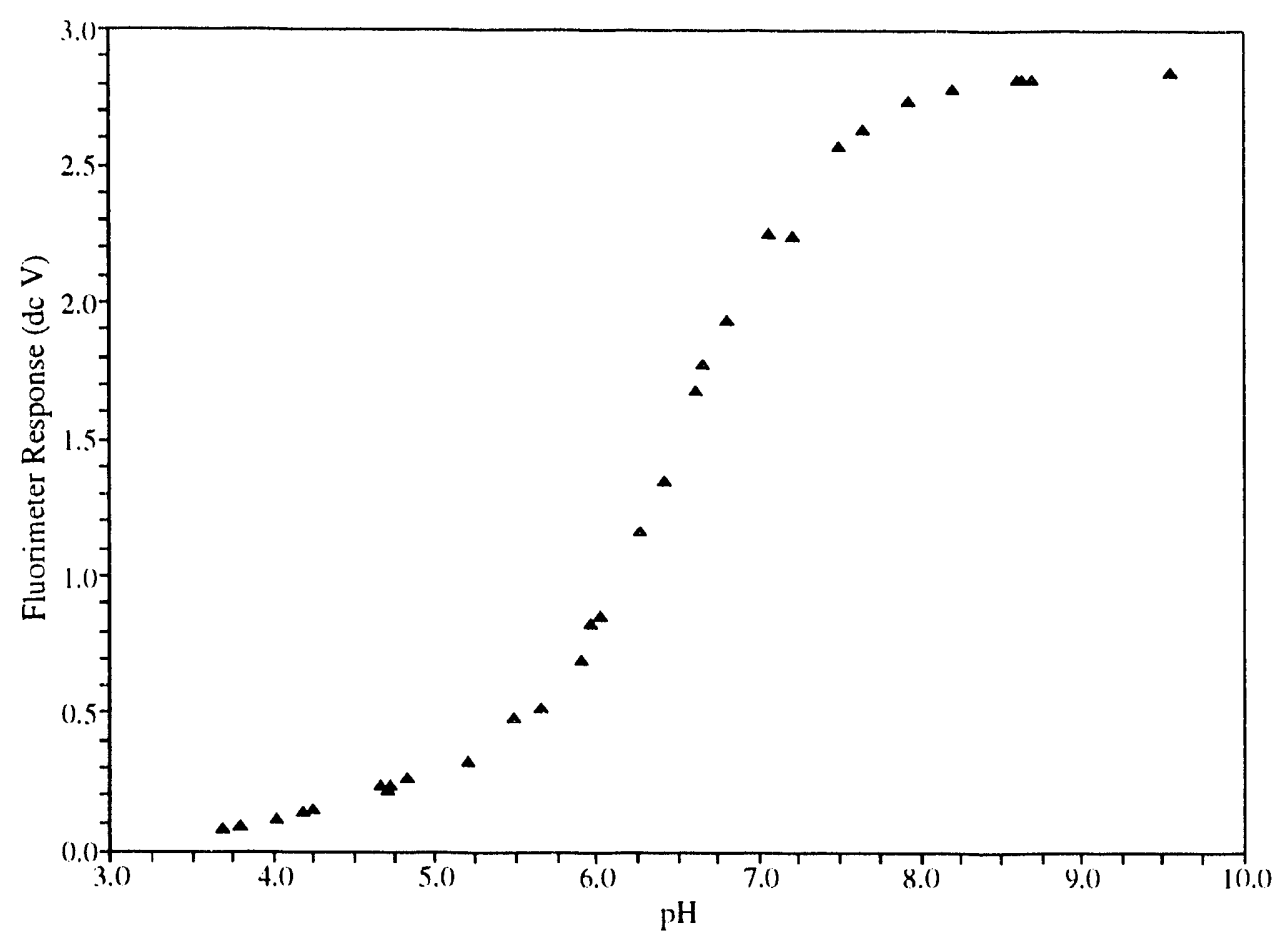

Figure 3.5: ('hange in fluorimeter response due to a change in pH for a constant dye concentration of $15 \mathrm{ppm}$.

response is affected when the $\mathrm{pH}$ varies. When a dc-voltage is impressed between the two electrodes, the resulting current flow induces a proton transfer reaction near the pulse wire. This reaction changes the $\mathrm{pH}$ of the solution immediately surrounding the wire. The application of a dr-voltage for a small interval of time, a pulse, hass created a small cylinder of fluid, a slug, that has an altered state and travels at the same velocity as the fluid in the column. As this slug travels downstream, it eventually passes in front of the optrode where the change in fluorescence, due to the altered $\mathrm{pH}$, is observed by the RFF. Due to dispersion, this phenomenon is observed as a breakthrongh curve. From this curve the velocity of the fluid can be calculated. A typical curve illustrating this result is shown later in Figure 4.6. The problem is to determine which is the best way to calculate the 
fluid velocity from the curve.

\subsection{Capillary Tube and Flow System}

The purpose of the capillary tube was to test the measurement method used in the porous media probes in a controlled system. Therefore, it was designed to enable the use of the same measurement parameters that were used in the probes. The tube and fiber mounting system were made with clear lucite plastic and were constructed in the University of Vermont machine shop. A fiber handling tool was used to hold the optical fiber and a depth finder was used to accurately position the fiber tip in the tube.

The inside diameter of the capillary tube is $4.76 \mathrm{~mm}\left(\frac{3}{16} \mathrm{in}\right)$ and the wall thickness is $1.59 \mathrm{~mm}\left(\frac{1}{16} \mathrm{in}\right)$. The tube length is $31.5 \mathrm{~cm}$ and the pulse wire is $13.4 \mathrm{~cm}$ from the inlet end of the tube. The distance between the pulse wire and the collector wire is $4 \mathrm{~cm}$. The distance between the pulse wire and the optical fiber can vary. The capillary tube has holes drilled in the side for the fiber at positions $0.0 \mathrm{~mm}, 3.0 \mathrm{~mm}, 6.0 \mathrm{~mm}, 9.0 \mathrm{~mm}, 12 \mathrm{~mm}, 20 \mathrm{~mm}$ and, $30 \mathrm{~mm}$ downstream of the pulse wire. The position at $3.0 \mathrm{~mm}$ matches the distance between the pulse wire and the optrode in the probes used in the porous medium. Because of time constraints, and because the scope of this investigation is limited to velocity experiments, the other downstream positions for the fiber were not used. If the scope of this investigation were extended to include a study about dispersion, then the other positions would be an integral part of the resulting experiments.

To insure capillary flow, the Reynolds number $\left(R_{e}\right)$ for the capillary tube with typical flow velocities was calculat $\cdot$. If the Reynolds number is in the laminar flow region, then it can be assumed that we have capillary type flow. For the flow velocities neighboring those in the column experiments $(\approx 0.10 \mathrm{~mm} / \mathrm{sec})$, a 
Reynolds mumber was calculated for the tube experiments using

$$
R_{r}=\frac{V d}{\nu}=\frac{\frac{Q}{A} r_{h}}{\nu}
$$

where $\frac{Q}{A}$ is the mean flow velocity of the fluid, $l$ is the kinematic viscosity and $r_{h}$, the hydraulic radius, is used for the characteristic length, $d$. The resulting Reynolds number of approximately 0.5 is well within the range for laminar flow $\left(R_{*}<10[\right.$ Bea79, pg. 65])

The apparatus that positions and holds the tube is constructed so that the position of the tube can be adjusted to level the tube and position it at a right angle to the fiber. The fiber can be moved to different positions along the tube. The mounting system for the fiber uses a depth finder apparatus (on loan from the UVM Engineering Machine Shop) which can adjust the depth of the tip of the fiber in the tube to an accuracy of $\pm 0.03 \mathrm{~mm}$ ( $\left.\frac{1}{1000}^{\text {th }} \mathrm{in}\right)$. The apparatus is shown in Figure 3.6.

The flow system for the capillary tube experiments is a simple system. The flow pump is a MasterFlex pump using size 13 MasterFlex tubing. The reservoir for the tracer dye is typically a $500 \mathrm{ml}$ Erlenmeyer flask with the inlet tube from the pump freely suspended in the Huid. During a typical velocity experiment, the capillary tube is saturated with the tracer dye before starting the experiment. The drain for the capillary tube has a $76 \mathrm{~mm}$ ( $3 \mathrm{in}$ ) length of tubing (the flow reducer) that is approximately twice the diameter of the capillary tube. The flow reducer prevents the siphon affect created if the end of the drain tubing is below the experiment tube. At the beginning of each experiment, the drain tube is connected to an empty $1 \mathrm{ml}$ pipette which is used with a stopwatch to determine the volumetric flow rate. 


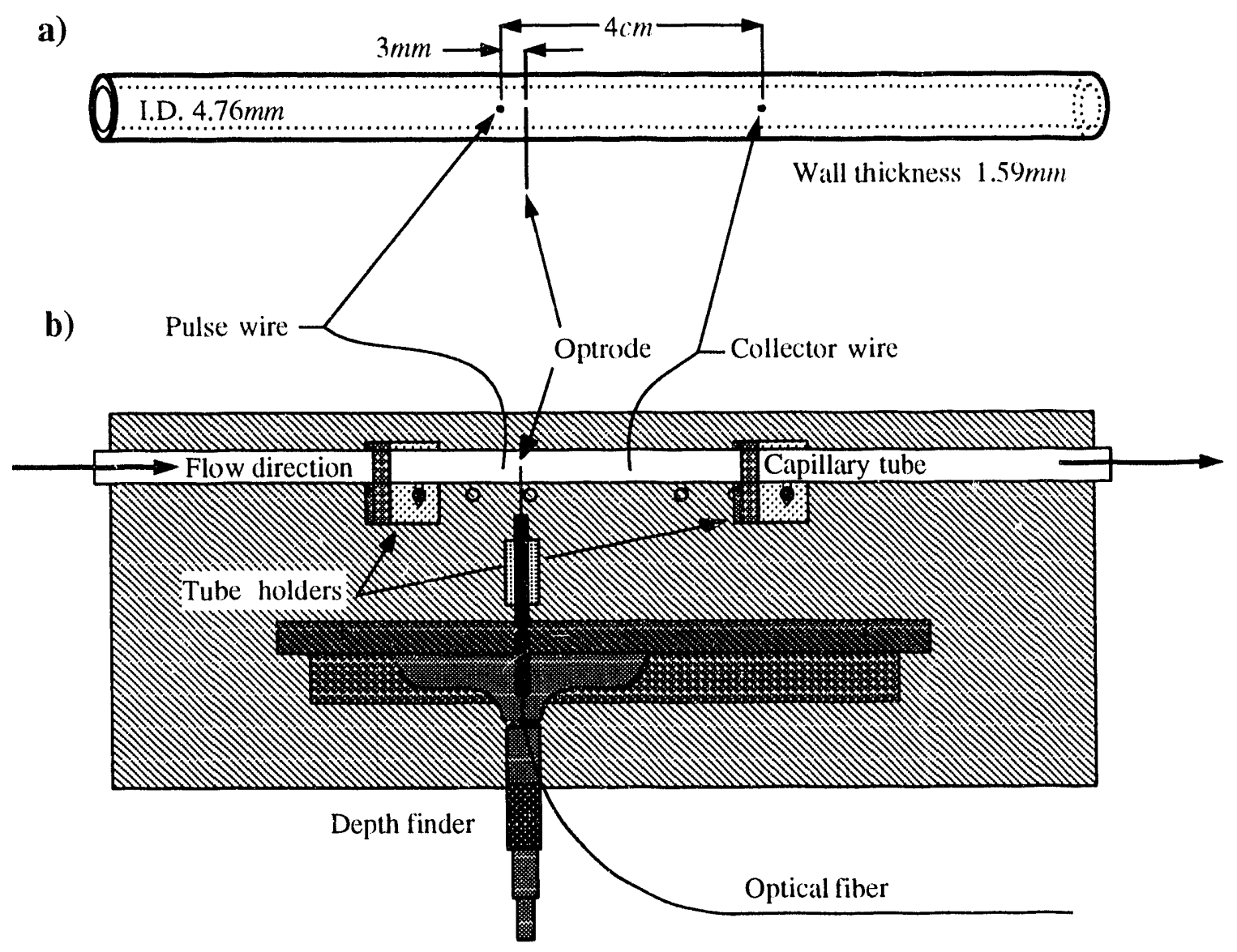

c)

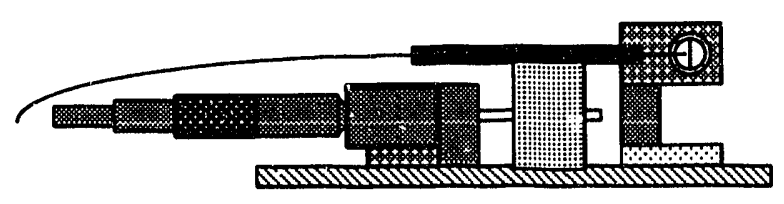

Figure 3.6: a) Capillary tube dimensions, b) top view capillary tube and holder, and c) side view capillary tube and holder. 


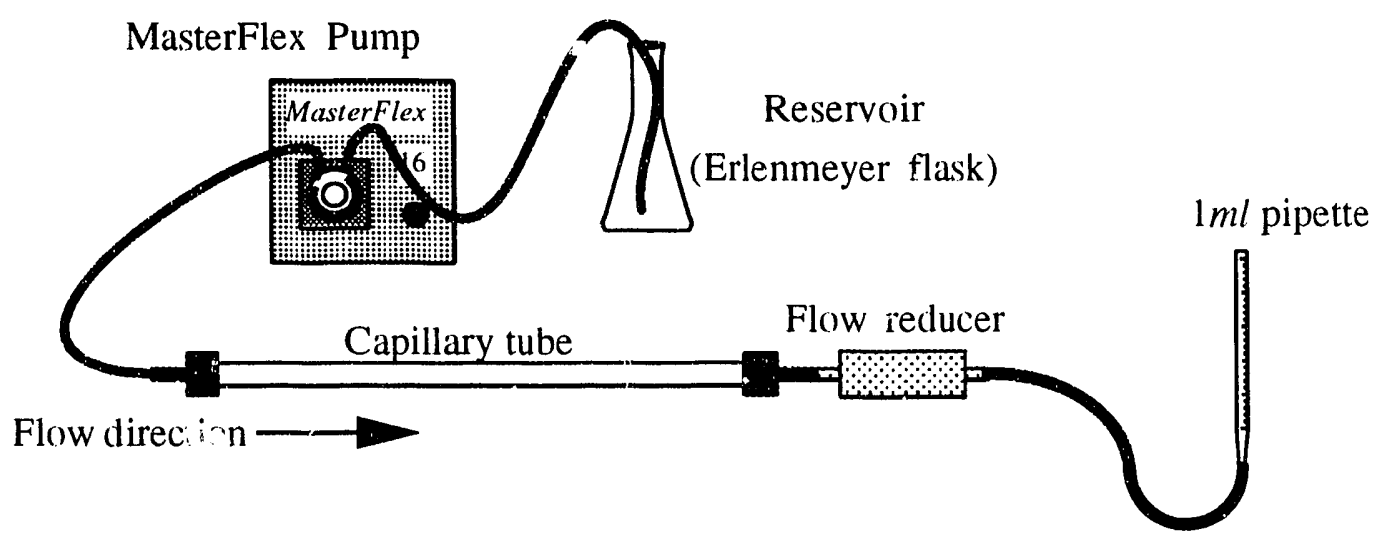

Figure 3.7: Flow system for capillary tube experiment.

\subsection{Experimental Procedure}

The experiments conducted in this research were designed to measure fluid veloc:ities under different flow rates and different pulse durations. TT.: experimental design consisted of thirty six experimental runs; Six at different flow rates and at each flow rate, six different pulse durations. One experimental run ronsisted of twelve repetitions; Each repetition at a constant volumetric flow rate and a constant pulse duration. This experimental design follows a six by six factorial analysis and enables a statistical analysis to be performed on the results.

it the onset of each run the air bubbles, if any, were cleared from the experiment tube, and the tracer solution was flushed through the tube at a high fluw rate. The flow rate was then decreased and adjusted to the nominal flow rate designation for the run. The exact flow rate is not known beforehand because the digitul readout on the MasterFlex pump is not accurate enough. Therefore, the flow rate is measured during each run.

A program, entitled VEL.PRG (see Appendix A) and written in the Lab ASYST programming language, was used to control the data acquisition and execution of the twelve repetitions for each experimental run. The parameters 
required by the program before each run were filename, number of repetitions for the particular run, pulse duration, and delay between repetitions. The pulse voltage and pulse current were set on the voltage source and remained constant for all of the experimental runs. After the parameters were entered, the program paused waiting for a signal to start the run.

The drain tubing, connecting the capillary tube and the $1 \mathrm{ml}$ pipette, was completely filled with fluid and connected to the end of the pipette. When the fluid meniscus reached the $0.00 \mathrm{ml}$ mark, the stopwatch and the program were started. During the run, the time and fluid meniscus position in the pipette were recorded manually in order to determine the flow rate. Usually, the time was recorded at tenths of a milliliter increments. After each of the twelve repetitions, the program saved the results in a spreadsheet format data file.

The data files were imported into Quattro Pro 4.0 (QPro, Borland Corp.). It became evident later that writing macro programs in QPro to analyze the results was too slow and cumbersome as the macros became more complex. Instead, QPro was used to convert all the files to ASCII format in order to be analyzed by another program. The files were then up loaded in ASCII format to the UNIX system where they were analized with a NAWK program. NAWK, a pattern scanning and processing language, bypasses the details of "data type" and "data input/output" protocols required in a conventional FORTRAN or (' program. NAWK was suitable in this situation because the program was small and only two operations needed to be performed: One operation to normalize the data, which involved subtracting the background readings from the data; the other to find the first arrival time. For each of the 432 files, the NAWK program would output to a file the name of the file and the first arrival time. The name of the file served as the index for the flow rate designation and the pulse duration. The 
results of the NAWK output were then downloaded to DOS and imported into QPro again in order to utilize the spreadsheet's lookup facility. A velocity was calculated for each of the first arrival times. The lookup facility was utilized to match the velocities derived from the NAWK program output with the database of velocities recorded during the experimental runs. Then, in the spreadsheet, the data files for SAS/STAT (a statistical software, SAS Institute, Inc.) were set up and up loaded again to the UNIX system to be analized with the SAS GLM (General Linear Models) Procedure.

Three preliminary experiments were conducted to study the effective path length of a bare ended polished fiber, the orientation of the optical fiber in relation to the pulse wire, and the characteristics of the fluid medium. For the effective path length experiments, the program described above had not been completed, so each repetition was started manually and the data was collected by an earlier version of the program. Since the velocity was not being considered, the flow rate was not measured. The depth finder apparatus mentioned in Section 3.4 was used to position the fiber tip at the center of the tube. Then, for each run, the tip was moved in increments of $0.5 \mathrm{~mm}$ away from the center line. Five different runs, each consisting of three repetitions were performed. The first run was conducted with the tip of the fiber at the center of the tube and the last one was conducted with tip at $2.0 \mathrm{~mm}$ from the center. The data files from these runs were imported into QPro and the results from each run were normalized, averaged, and plotted. These results are shown in Section 4.3.

The other two experiments were conducted manually by observing the response and recording the value from the multimeter by hand. Again, for these experiments the flow rate was not needed and, therefore, not recorded. 


\section{Chapter 4 Discussion of Experimental Results}

\subsection{Introduction}

The velocity probe proposed by Turan had a number of design variables that needed to be investigated before the central goal of this thesis research could be pursued. These variables included the location of the tip of the fiber relative to the center of the pore, the orientation of the fiber relative to that of the pulse wire, the duration of the pulse, and the characteristics of the fluid medium used in the experiments. The results of these investigations are presented in the following sections. The experiments dealing with the duration of the pulse were combined with the velocity experiments and a relationship has been developed between the volumetric flow rate velocity, termed theoretical velocity $\left(V_{t}\right)$, and the velocity calculated from the response measurements taken with the fiber sensor, termed measured velocity $\left(V_{m}\right)$. The pulse duration was also found to be significant in this relationship. A statistical analysis of the data is discussed in the last section which presents the results of the analysis, the model used in the analysis and the significance of each of the independent variables used in the model. 


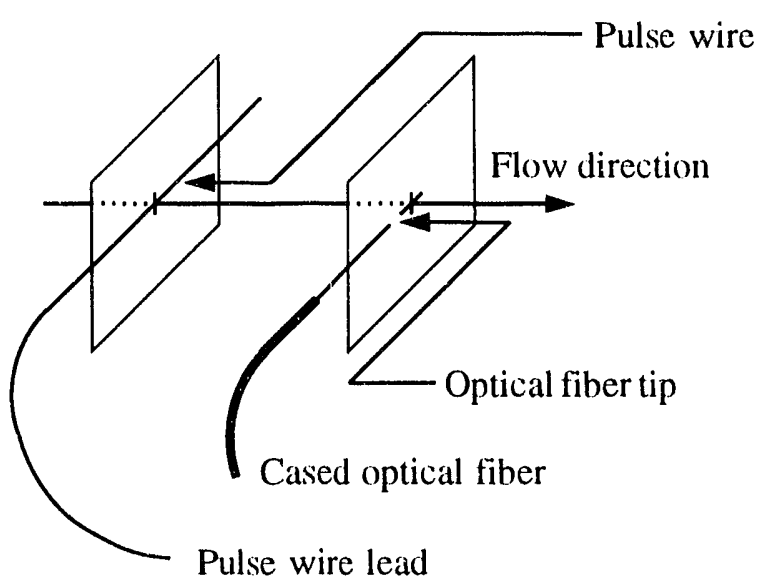

(a) Parallel orientation

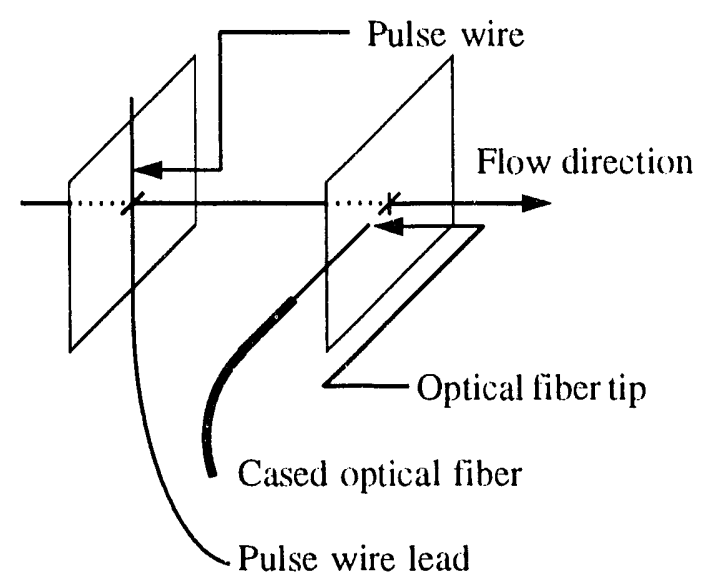

(b) Perpendicular orientation

Figure 4.1: Two investigated orientations of the optical fiber relative to the pulse wire. (a) Parallel orientation and (b) perpendicular orientation.

\subsection{Orientation Experiment}

The first design to implement the velocity measurement method in a tube resembled the probe first proposed by Turan (see Figure 1.3). One variable in this design was the orientation of the optical fiber relative to the pulse wire. The possible orientations were somewhat limited by the cubic structure of the probes, the criteria that the probes have to be reproducible, and the fact that the construction of the probes is difficult. Turan's proposed probe had a parallel orientation in which the direction of the optical fiber is parallel to the direction of the pulse wire; this type of orientation is illustrated in Figure 4.1(a). This orientation was found to be ineffective because of the size of the slug created around the pulse wire by the pulse. The slug is so small that, if the stream lines in which it was created do not pass directly in front of the fiber tip, then no effect will be measured by the RFF. The solution to this problem was to change the orientation, as shown in Figure 4.1(b), so that the optical fiber was perpendicular relative to the pulse wire. This orientation created a situation in which the slug would pass as a 


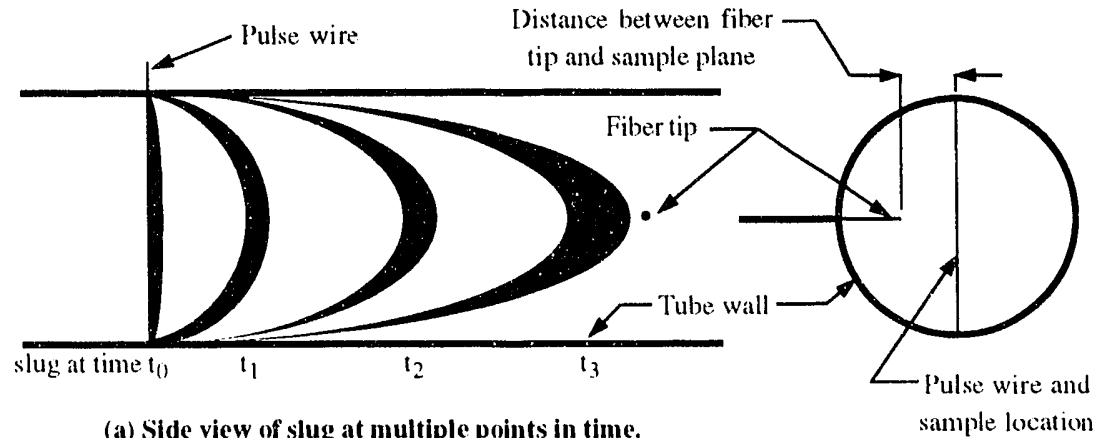

(a) Side view of slug at multiple points in time.

(b) Front view

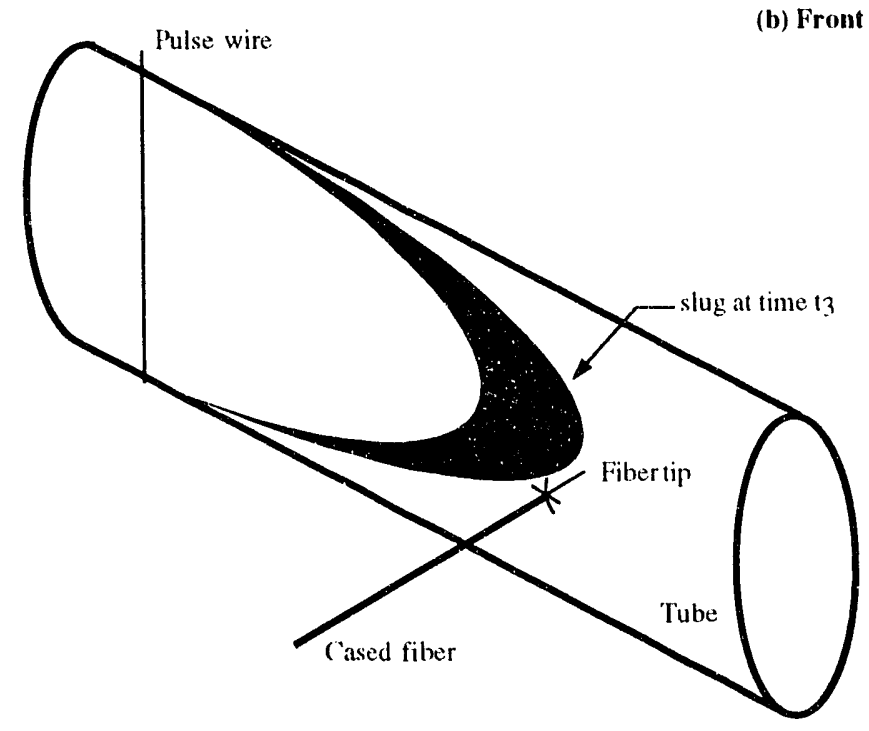

(c) Perspective view of slug at time $t_{3}$

Figure 4.2: (a)Side view, (b)front view, and (c)perspective view of slug in the shape of a planar parabola in the tube.

crescent with the ends at the top and bottom of the pulse wire (see Figure 4.2). The optical fiber would observe only a slice through the tip of the parabola.

The perpendicular orientation produced results that were more consistent and repeatable, and led to a change in the design of the probes constructed by Turan. The revised probe, as a result of this first experiment, is shown in Figure 4.3. 


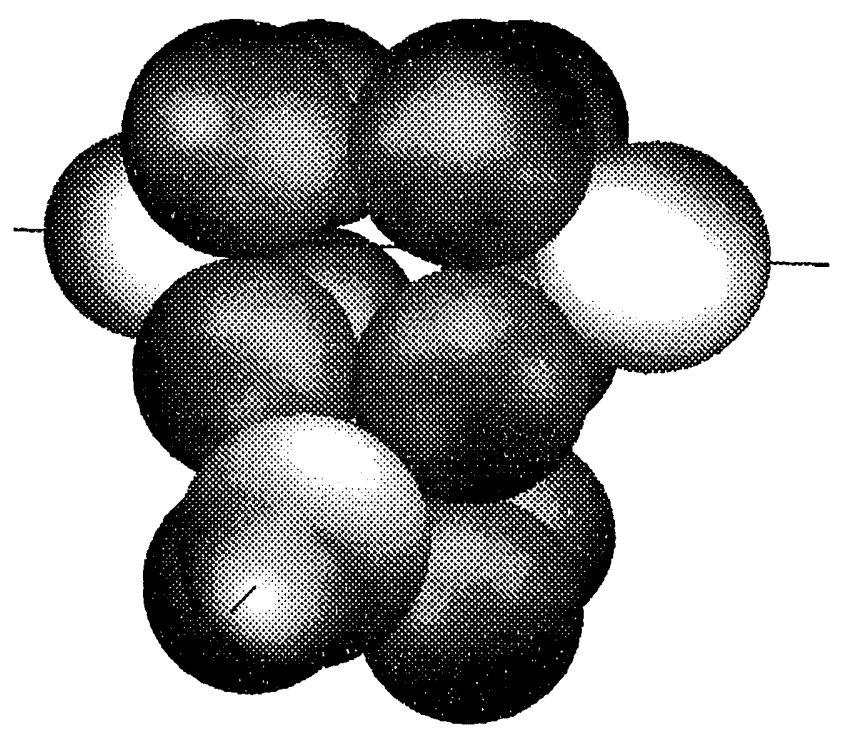

Figure 4.3: The revised velocity probe used in future porous media experiments by Turan.

\subsection{Radial Position of Fiber Tip}

The fiber optrode samples the region directly in front of it, as described by the effective path length illustrated in Figure 3.4. The distance between the fiber tip and the sample plane (see Figure $4.2(b)$ ), which by design is at the center of the pore, is another design variable of concern. An experiment was performed in the (apillary tube to study the effective path length of a bare-ended polished fiber. The objective for this study was to determine whether or not the fiber tip could be positioned away from the center of the pore in order to avoid obstructing the flow. From this experiment, it was found that the fiber tip should be positioned at the center of the pore.

The experiment to study the effective path length was performed for live different fiber tip positions, with three repetitions at each location. The average of the three repetitions at each fiber tip position is plotted in Figure 4.4 . The RFF response readings were normalized, before being plotted, by subtracting the 


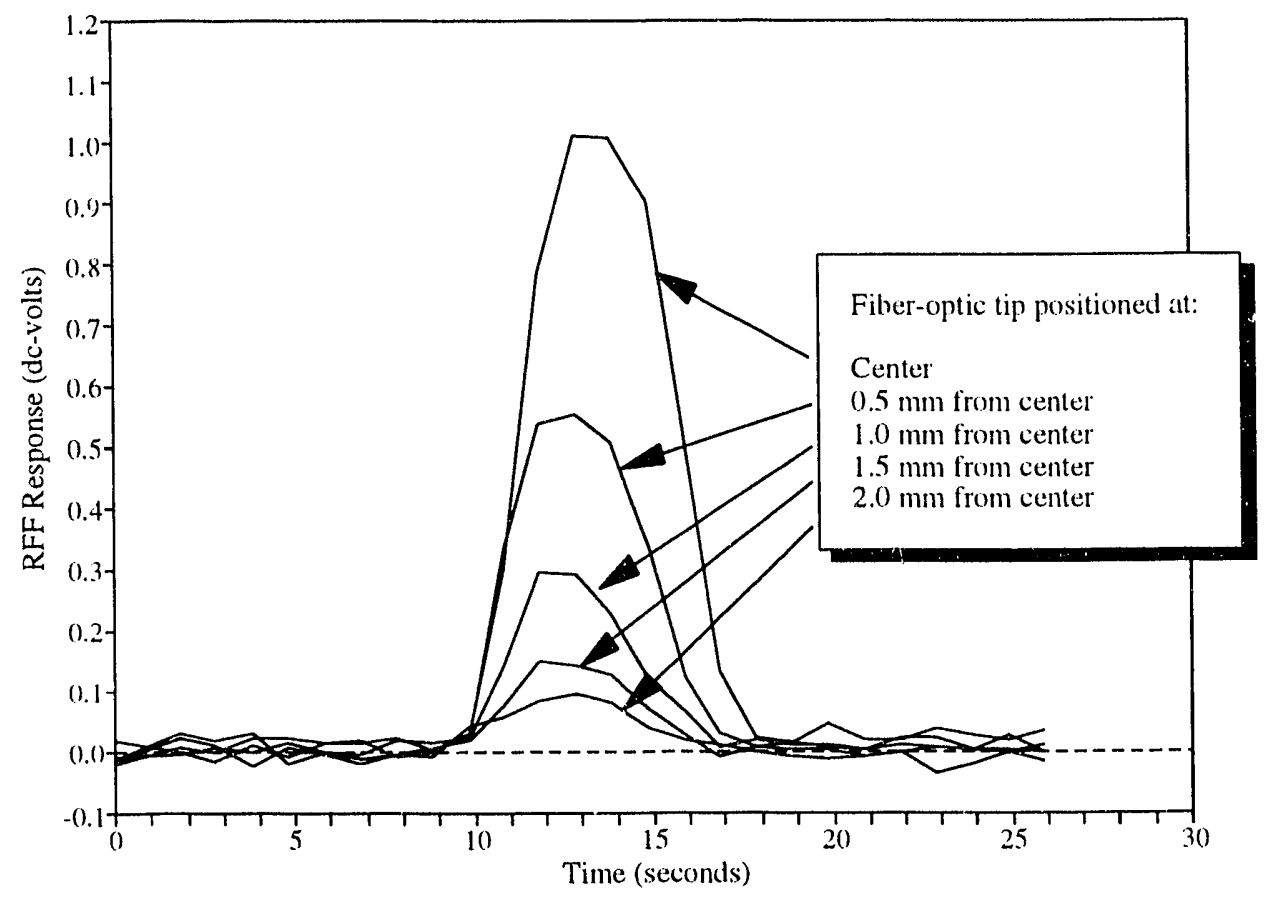

Figure 4.4: Effect of sample distance from fiber tip. Each curve is the average of three repetitions at that distance. The solute concentration was $10 \mathrm{ppm}$ and the pulse duration was $1.0 \mathrm{sec}$. The RFF response was normalized by subtracting the background readings from the measurements. 
background readings from the response measurements. The concentration of the tracer for this experiment was $10 \mathrm{ppm}$ and the pulse duration was one second.

The results of this study show that the distance from the sample plane to the fiber tip has an effect on the RFF response. It can be seen in Figure 4.4 that moving a distance of only $0.5 \mathrm{~mm}$ away from the sample causes a $45 \%$ decrease in RFF response.

From this study, it has been determined that the best position for the fiber tip is at the center of the pore, or as close as possible to the slug being sampled. Because of this result, all future probes were constructed with the fiber tip positioned at the center of the pore.

\subsection{Characteristics of the Fluid Media}

As was mentioned in Section 3.3, the response measured by the RFF is partly dependent on the $\mathrm{pH}$ of the fluid. An experiment was performed to determine a general relationship between the $\mathrm{pH}$ of the fluid and the response measured by the RFF. Figure 3.5 is repeated here to illustrate the results of this experiment.

From this experiment it was found that as the $\mathrm{pH}$ increases, so does the response measured with the RFF. Also, it was found that the tracer tended to favor a neutral $\mathrm{pH}(\mathrm{pH}$ of 6.5$)$. In other words, the pulse would only cal se a change in the $\mathrm{pH}$ up to a $\mathrm{pH}$ of 6.5 . At a $\mathrm{pH}$ of 6.5 , there was almost no measurable effect caused by the pulse. The same is true for the reverse when starting at a higher $\mathrm{pH}$ and reversing the polarity.

For all of the capillary tube experiments the pH was adjusted to 5.0 so that a pulse in the fluid would cause up to a one volt-de increase in RFF response. 


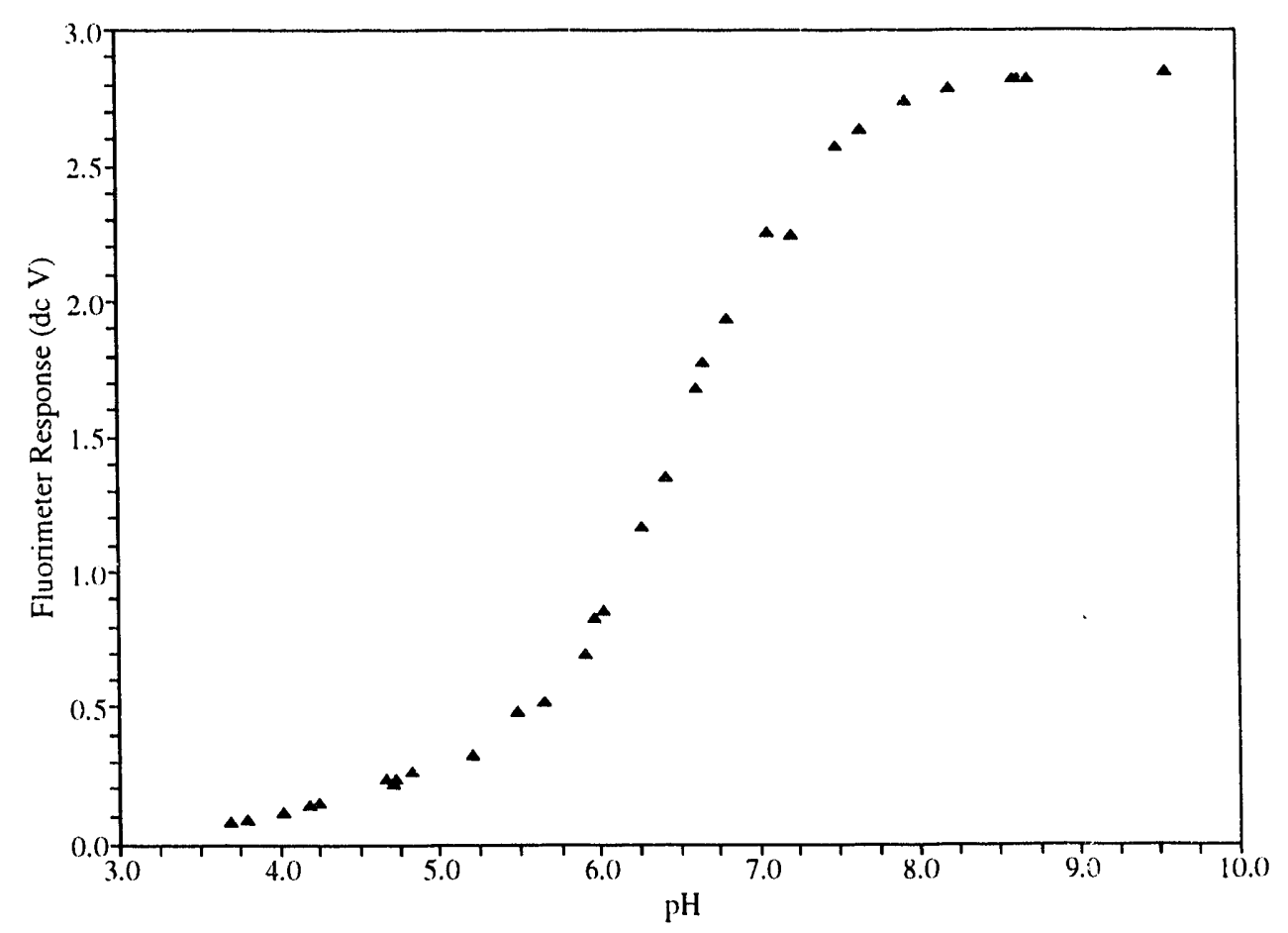

Figne 4.5: (hange in fluorimeter response due to a change in $\mathrm{pH}$ for a constant dye concentration of $15 \mathrm{ppm}$.

\subsection{Velocity and Pulse Duration Experiments}

The pulse duration and velocity of the fluid were the parameters of interest when determining the relationship between measured velocity and theoretical velocity. Both the pulse duration and the fluid velocity tended to have a scaling effect on the response-time curve that was produced from the experiments. In order to quantify this effect, an experiment was performed for six different pulse durations and six different flow rates. For each combination of pulse duration and flow rate, twelve repetitions were performed. The flow rate was measured once during each run; a run being twelve repetitions at the same pulse duration and flow rate. There were 72 different repetitions (six runs) for each flow rate. The flow rate was 
Table 4.1: Maximum Volumetric Flow Rate Velocities

\begin{tabular}{|c|cccccc|}
\hline Flow Designator & \multicolumn{5}{|c|}{ Pulse Duration (seconds) } \\
& 0.25 & 0.50 & 0.75 & 1.00 & 1.50 & 2.00 \\
\cline { 2 - 7 } & \multicolumn{6}{|c}{ Max Velocity $(\mathrm{mm} / \mathrm{sec})$} \\
A & 0.308 & 0.305 & 0.310 & 0.316 & 0.312 & 0.313 \\
B & 0.238 & 0.235 & 0.236 & 0.235 & 0.237 & 0.235 \\
( & 0.194 & 0.192 & 0.196 & 0.200 & 0.197 & 0.192 \\
D & 0.152 & 0.153 & 0.150 & 0.157 & 0.153 & 0.153 \\
E & 0.119 & 0.120 & 0.119 & 0.125 & 0.125 & 0.125 \\
F & 0.099 & 0.099 & 0.099 & 0.090 & 0.110 & 0.108 \\
\hline
\end{tabular}

found to vary slightly from run to rum. This variation is illustrated in Table 4.1, which is a table of the maximum velocities calculated from the flow rate data measured manually during each rum.

The variation in flow rate over the six pulse duration runs is due to the speed control of the pump. With time, the speed of the pump tends to vary slightly. The maximum variance over six runs at the same flow designation is $5.26 \times$ $10^{-5}(\mathrm{~mm} / \mathrm{sec})^{2}$ which occurs at the lowest flow.

Figure 4.6 is a typical plot of fluorimeter response versus time. The first arrinal time, labeled on the plot, is the travel time of the slug of fluid, created by the pulse, from the pulse wire to the optrode. The label first arrinal time is used because it is the time that the front of the slug is first recognized by the optrode. This time is determined by evaluating a moving gradient along the curve. The first arrival time is defined as the time when the gradient of five adjacent points on the curve is greater than five percent. Thus, the measured velocity of the fluid is

$$
V_{m}=\frac{\text { distance between pulse wire and optrode }}{\text { first arrival time }}[\mathrm{mm} / \mathrm{sec}] \text {. }
$$

The velocities in Table 4.1 were paired with the velocities calculated using the 


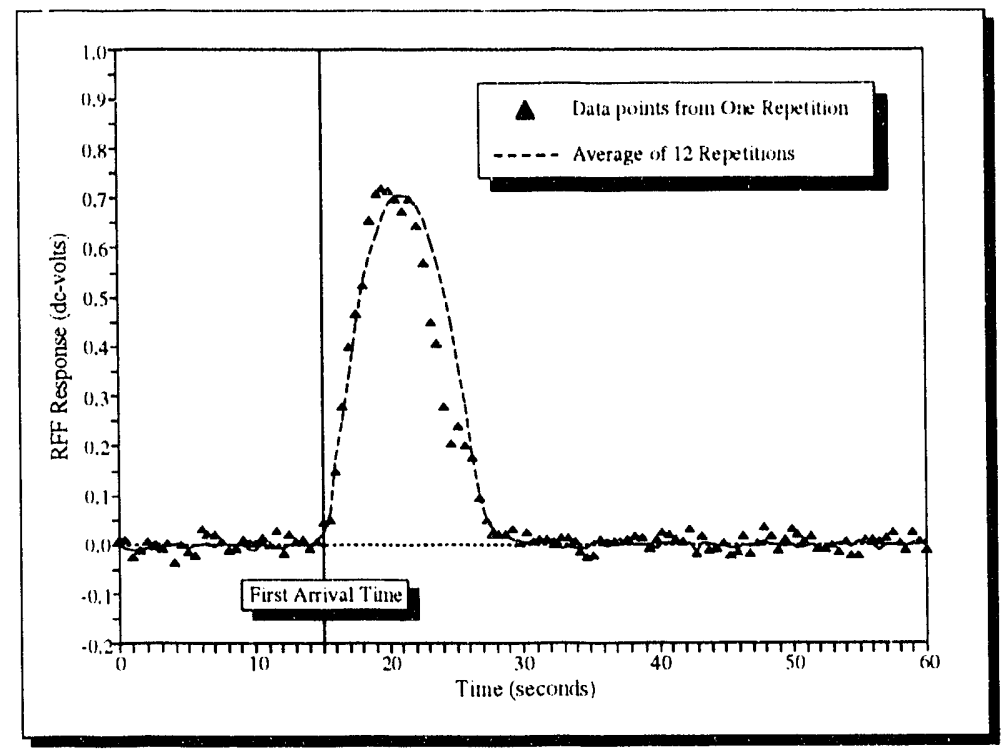

Figure 4.6: Example of response curve produced from the experimental data of a run with Flow Designation (' and Pulse Duration 1.50 scconds. The volumetric flow rate velocity, from Table 4.1 , is $0.197 \mathrm{~mm} / \mathrm{sec}$, compared to $0.199 \mathrm{~mm} / \mathrm{sec}$ calculated from the First Arrival Time of 15.07 seconds.

first arrival time. A linear multiple regression analysis was performed on this data set resulting in the relationship

$$
V_{t}=1.07 V_{m}-0.014 P
$$

where $V_{t}$ is the theoretical velocity, $V_{m}$ is the measured velocity calculated using first arrival times, and $P$ is the pulse duration.

This relationship, along with the normalized data, is shown in Figures 4.7 through 4.13 for all pulse durations and flow rate combinations. The statistical significance of the results is discussed in the next section.

\subsection{Statistical Analysis}

The results presented in the previons section show that a relationship between the theoretical velocity and the first arrival time velocity, or measured velocity, 


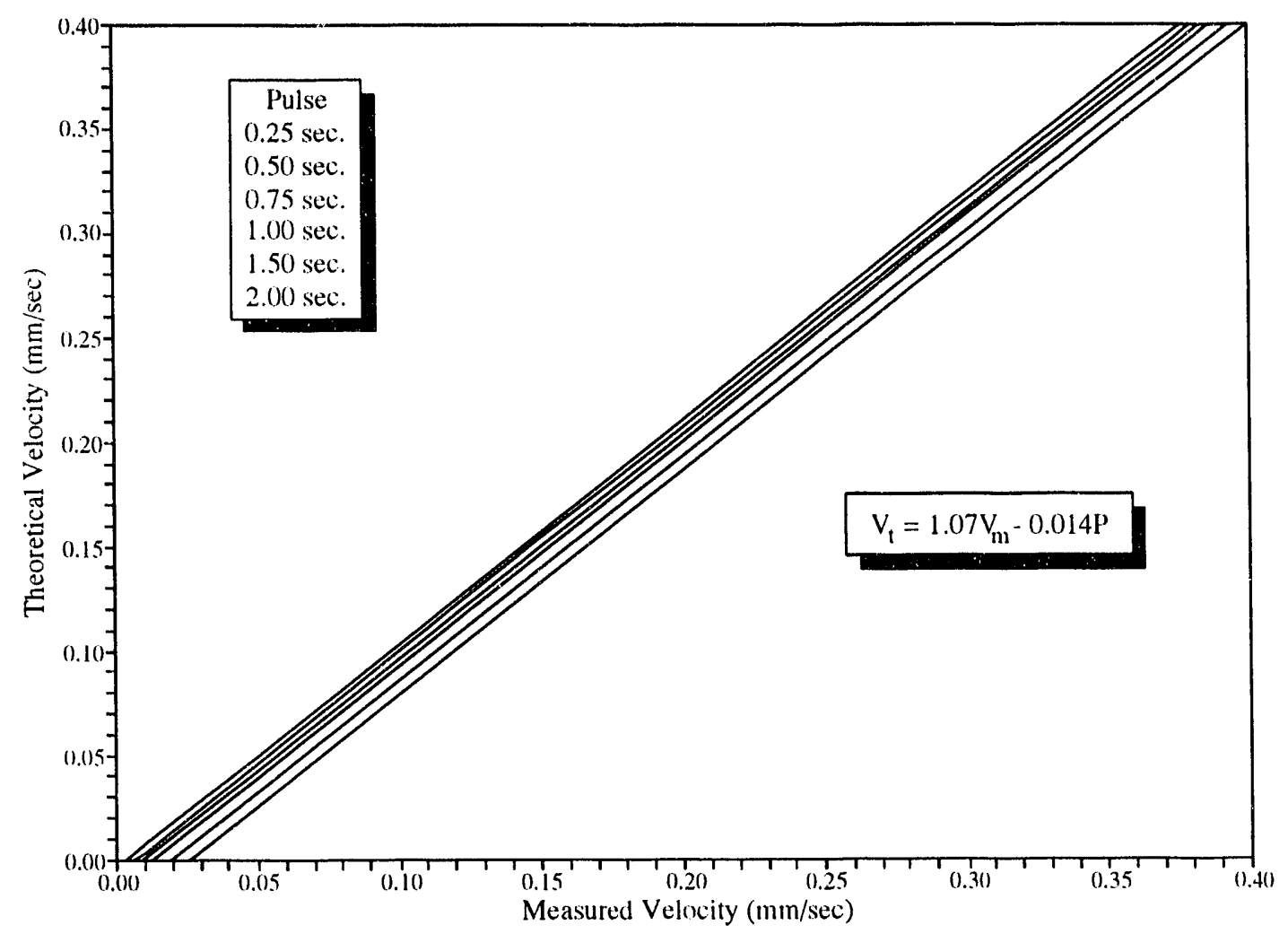

Figure 4.7: Relationship for all six pulse durations. From top to bottom $P^{\prime}=$ $0.25 \mathrm{sec}$ to $2.00 \mathrm{sec}$. 


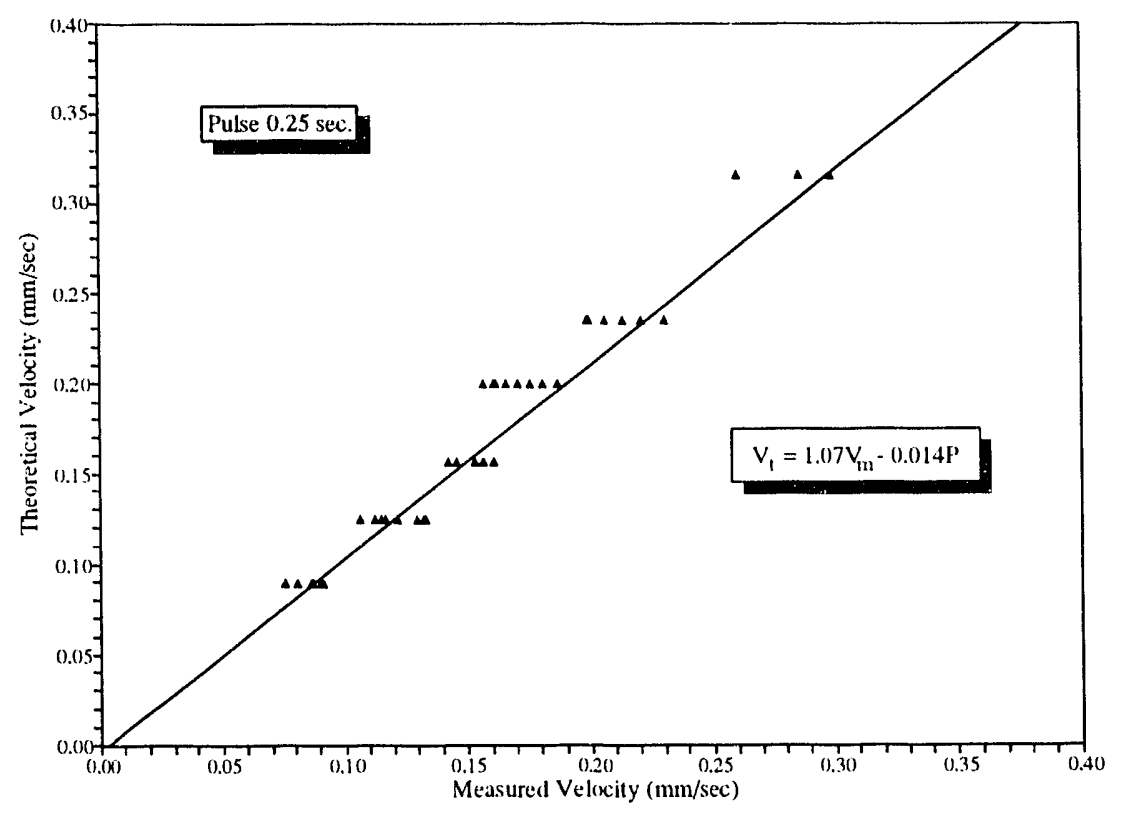

Figure 4.8: Result for a pulse duration of 0.25 seconds.

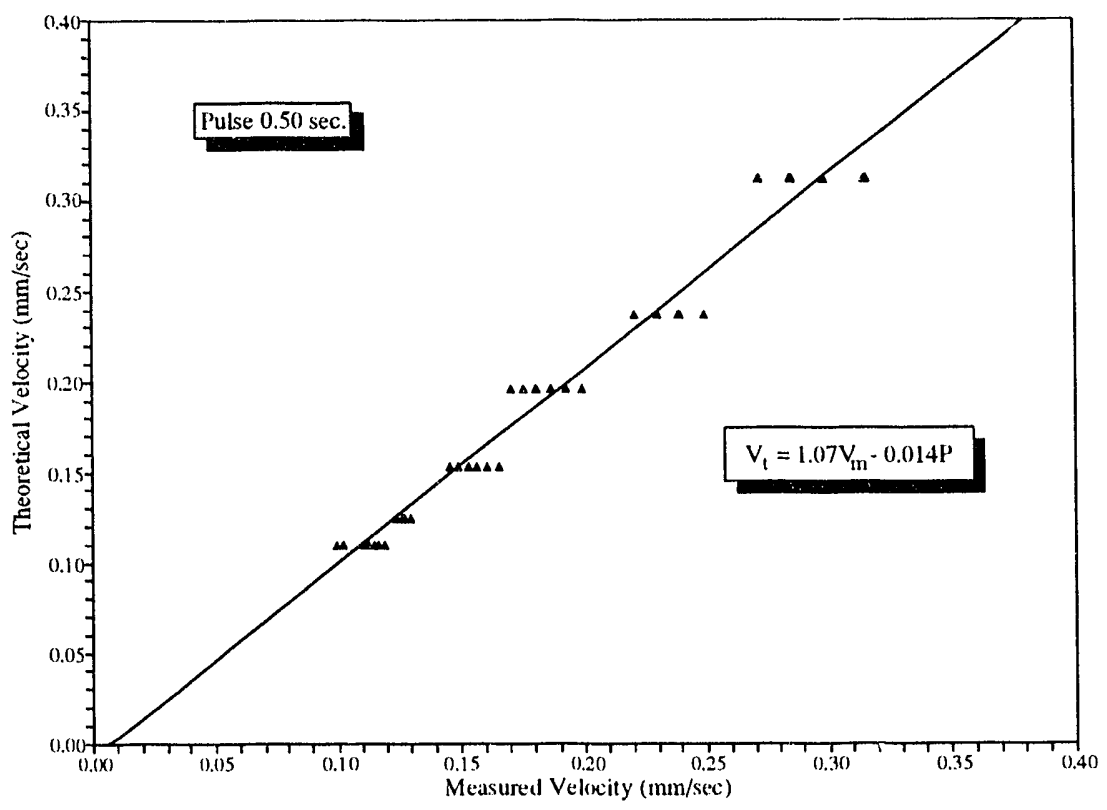

Figure 4.9: Result for a pulse duration of 0.50 seconds. 


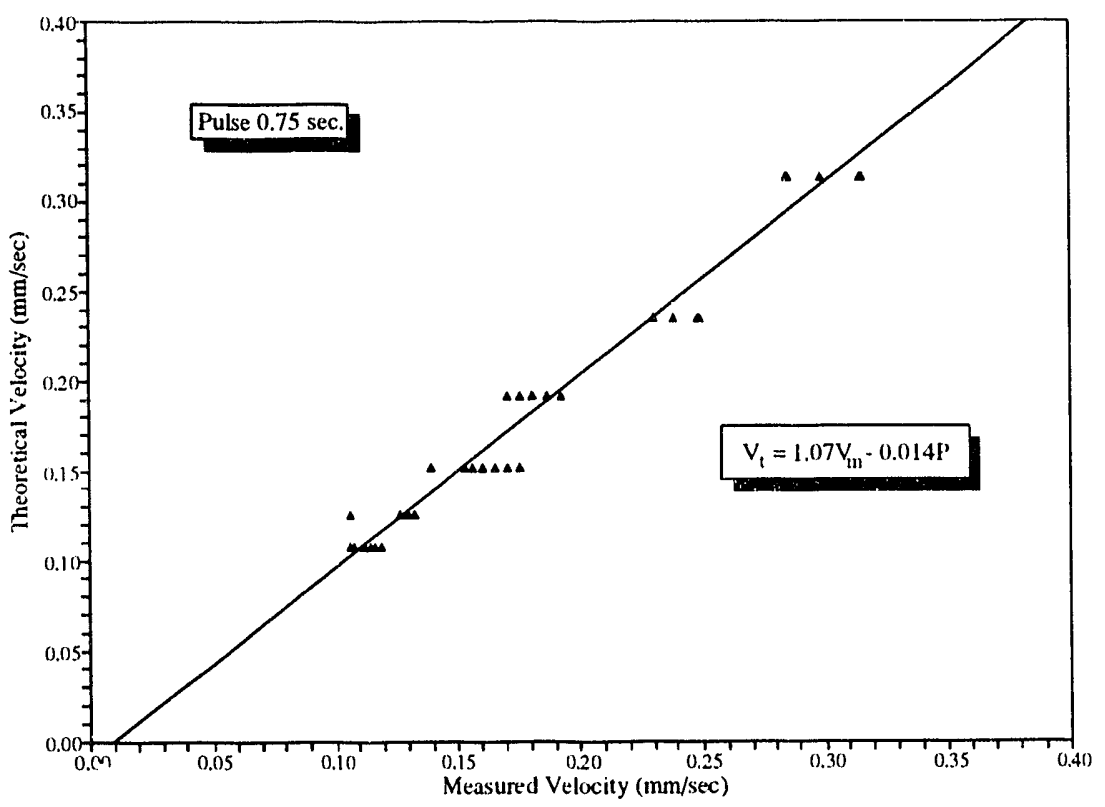

Figure 4.10: Result for a pulse duration of 0.75 seconds.

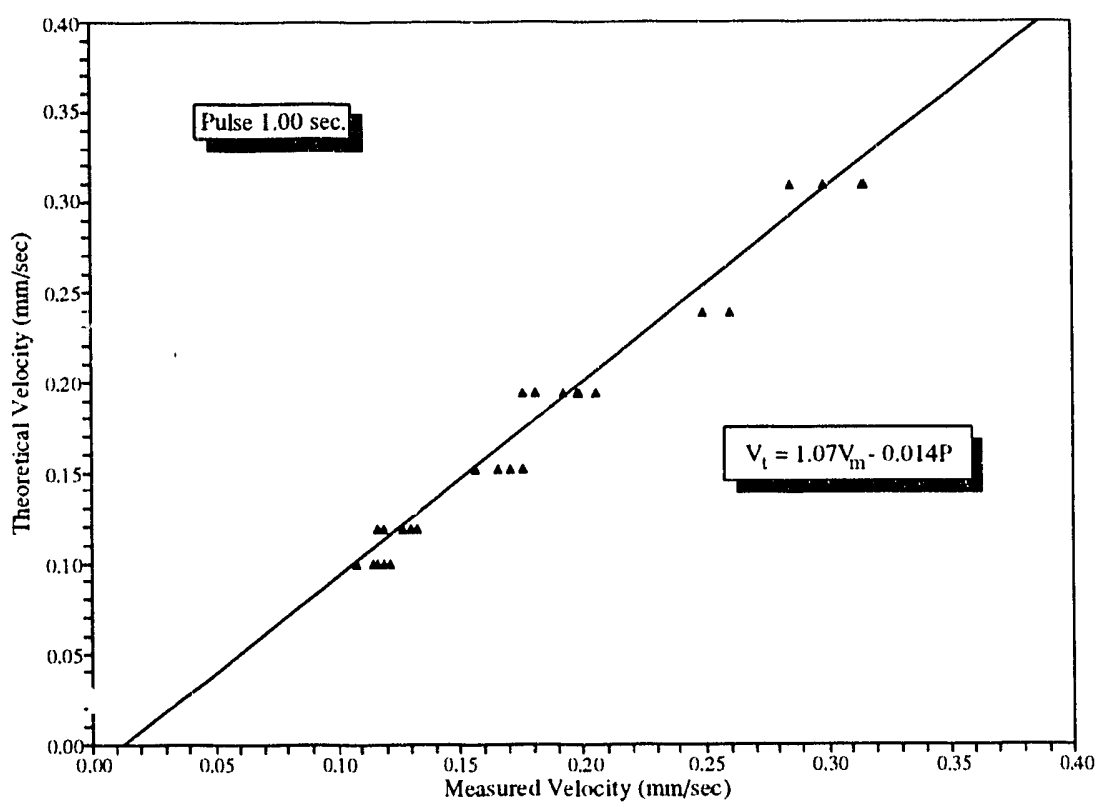

Figure 4.11: Result for a pulse duration of 1.00 seconds. 


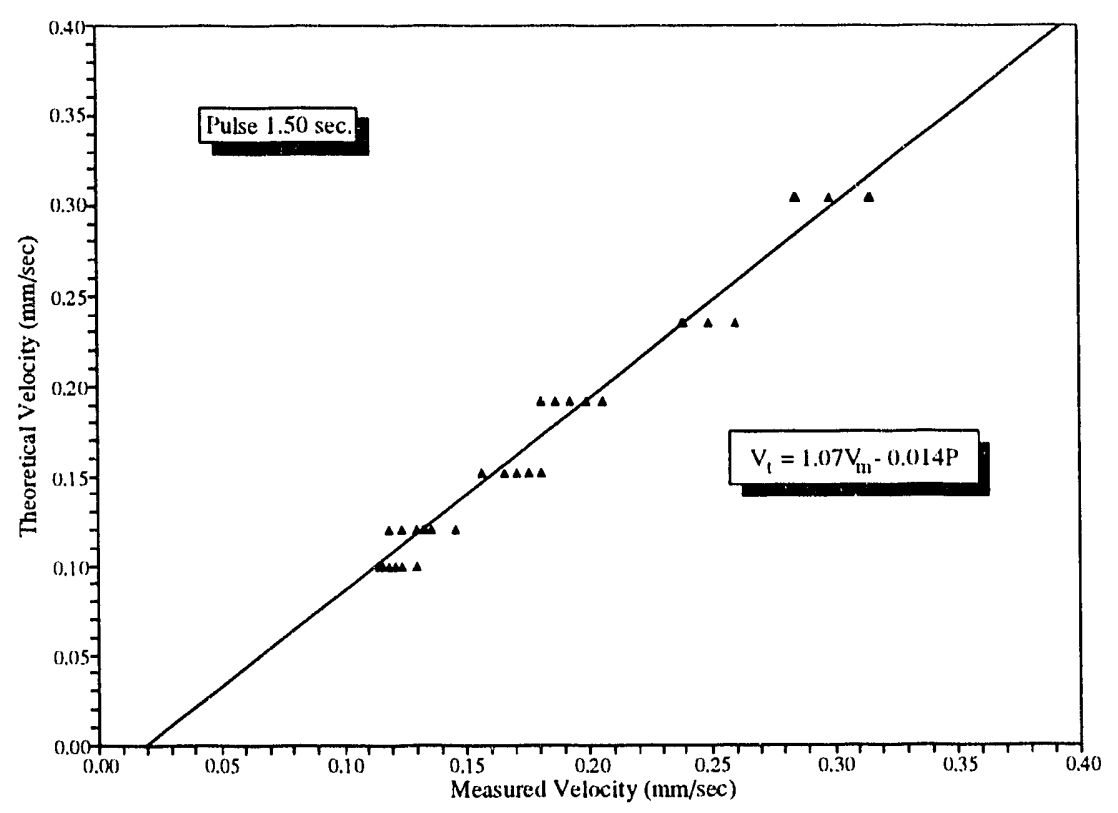

Figure 4.12: Result for a pulse duration of 1.50 seconds.

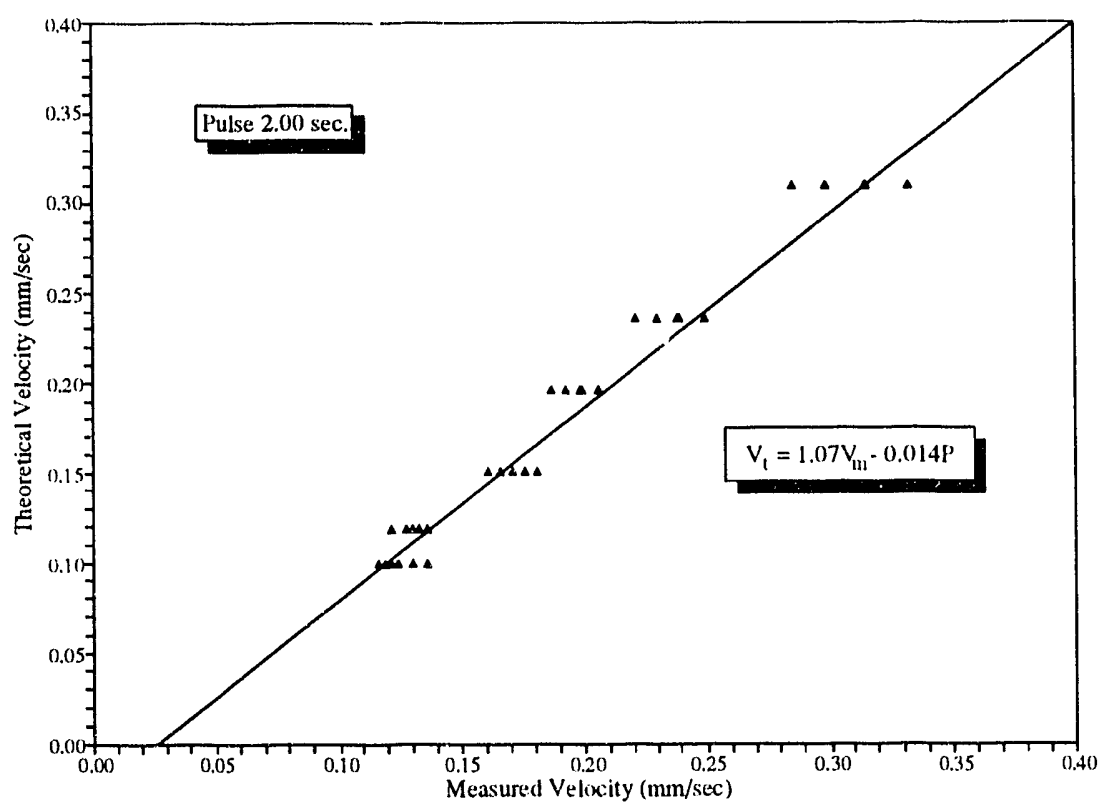

Figure 4.13: Result for a pulse duration of 2.00 seconds. 
Table 4.2: SAS Output With Interaction Term

\begin{tabular}{|c|c|c|c|c|}
\hline \multicolumn{5}{|c|}{$\begin{array}{c}\text { The SAS System } \\
\text { 18:15 Monday, November 16, } 1992 \\
\text { C.ieneral Linear Models Frocedure } \\
\text { Number of observations in data set }=432\end{array}$} \\
\hline \multicolumn{5}{|c|}{ Dependent Variable: VELT } \\
\hline Source & L.JF & Type I SS & F Value & $F^{\prime} r>F$ \\
\hline VELM & 1 & 2.102 & 1199 & 0.0001 \\
\hline FILSSE & 1 & 0.02981 & 170.0 & 0.0001 \\
\hline VELM*F'lLSE & 1 & 0.0001257 & 0.7200 & 0.3976 \\
\hline
\end{tabular}

does exist. In order to determine the coefficients of the equation given, and their significance, a statistical examination was performed on the data. This examination included an analysis of variance and a regression analysis. The combination of these two analyses has provided a quantifiable relationship with an associated confidence level, rather than an inferred relationship.

The statistical design of these experiments is known as a factorial analysis of variance where measured velocity (VELM) and pulse duration (PULSE) are the parameters being considered. A factorial design is one which considers all possible interactions between the independent variables and the variance caused from changes in these variables. The result of this type of analysis is a measure of the influence that a given parameter or combination of parameters imparts on the dependent variable. In this situation the statistical model is represented by

$$
V E L T=V E L M+P U L S E+V E L M * P U L S E+C O N S T A N T
$$

where $V E L T$ is the theoretical, or volumetric flow rate velocity, and $V E L M *$ PULSE is the interaction between the pulse duration and measured velocity parameters.

These experiments were performed at six different flow rates and six different pulse durations which resulted in a $6 \times 6$ factorial design. The CLM (General 
Table 4.3: SAS Output Without Interaction Term

\begin{tabular}{|c|c|c|c|c|c|}
\hline \multicolumn{6}{|c|}{$\begin{array}{c}\text { The SAS System } \\
\text { 22:08 Tuesday, ()ctober 16, } 1992 \\
\text { (ieneral Linear Models Frocedure } \\
\text { Number of observations in data set }=4: 32\end{array}$} \\
\hline \multicolumn{6}{|c|}{ Dependent Variable: VELT } \\
\hline Source & D)F & $\begin{array}{l}\text { Sum of } \\
\text { Squares }\end{array}$ & $\begin{array}{l}\text { Mean } \\
\text { Square }\end{array}$ & F Value & $\mathrm{F} r>F$ \\
\hline Model & 2 & $2.1: 32$ & 1.066 & 6084.43 & 0.0001 \\
\hline Error & 429 & 0.07516 & 0.0001752 & & \\
\hline \multirow[t]{3}{*}{ Corrected Total } & $4: 31$ & 2.207 & & & \\
\hline & & R-Square & Root MSE & VELT Mean & \\
\hline & & 0.9660 & 0.01324 & 0.1863 & \\
\hline Source & [)F & Type ISS & F Value & $\mathrm{Pr}>\mathrm{F}$ & \\
\hline VELM & 1 & 2.102 & 1200 & 0.0001 & \\
\hline PULSE & 1 & 0.02981 & 170.2 & 0.0001 & \\
\hline P'arameter & & Estimate & $\begin{array}{c}\text { T for } \mathrm{HO}: \\
\text { P'arameter }=0\end{array}$ & $\mathrm{Tr}>|\mathrm{T}|$ & $\begin{array}{c}\text { Std Error of } \\
\text { Estimate }\end{array}$ \\
\hline INTERC:EF'T & & -0.001298 & -0.61 & 0.5416 & 0.002124 \\
\hline VELM & & 1.070 & 110.3 & 0.0001 & 0.009702 \\
\hline PULSE & & -0.01403 & -13.04 & 0.0001 & 0.001075 \\
\hline
\end{tabular}

Linear Models) Procedure of the SAS/STAT package was used to perform a statistical analysis of the results and to estimate the parameters for the model. The results of this examination are given in Tables 4.2 and 4.3 . Table 4.2 gives the SAS ontput with the interaction term $(V E L M * P U L S E)$ included in the model. From the $F$ statistic ( $F$ Value in the table), it is shown that interaction between measured velocity and pulse duration does not have a significant effect on the model. This is shown with a $60.2 \%$ confidence $(1.00-P r>F(0.3976))$. This outcome was expected but was checked in order to be statistically complete.

Table 4.3 gives the SAS output without the interaction term included in the model. The R-squared value indicates that $97 \%$ of the variation in the input is accounted for by the the model. The Parameter list at the bottom of the table gives the estimates (Estimate) of the coefficients for the relationship presented in the previous section. From the Student's $t$ value ( $\mathbf{T}$ for $\mathbf{H}_{0}$ :) for testing the null hypothesis and the adjacent column $(\operatorname{Pr}>|\mathbf{T}|)$, it is shown that the Intercept parameter is zero. In the column labeled $\operatorname{Pr}>|\mathbf{T}|$, a value of .54 
indicates that there is a $54 \%$ probability that a larger Student's $t$ value would result if the intercept were zero. A very small probability in this column leads to the conclusion that the particular independent variable contributes significantly to the model. Therefore, since the significance of the VELM and PULSE is by far greater than that of the INTERCEPT term, it can be neglected and treated as zero. 


\section{Chapter 5 \\ Conclusions and Future Research}

\subsection{Summary and Conclusions}

The complex velocity probe proposed by Turan has been investigated. Velocity measurements taken with the probe were found to be dependent not only on the velocity of the fluid, but also on other parameters. The parameters investigated in this thesis were the location of the tip of the optical fiber relative to the center of a pore, the orientation of the fiber relative to that of the pulse wire, the duration of the pulse, and the characteristics of the fluid used in the experiments.

A summary of the investigations of the parameters follows:

- The first experiment performed discovered a problem with the orientation of the fiber relative to that of the pulse wire in the proposed probe. This orientation was parallel (see Figure 4.1) in the proposed probe. It was discovered that a perpendicular orientation would produce results that were more consistent and more accurate.

- The radial distance of the fiber tip from the center of the tube was investigated to determine an actual effective path length (see Figure 3.4 and equation (3.2)) of a bare-ended polished fiber. The $L_{E}$ for the fibers used was found to be less than $1.0 \mathrm{~mm}$, which is half the previously suggested $L_{E}$ of $2 \mathrm{~mm}$. This result was shown in Figure 4.4 . 
- The response measured by the fiber fluorimeter was determined to be dependent on the concentration of the fluid and also on the $\mathrm{pH}$ of the slug created in the fluid by the pulse (see Figure 3.5).

- By comparing the response-time curves, it was determined that the best point on the curve from which to determine the velocity is the first arrival time. This was defined simply as the travel time of the slug measured when the front of the slug first reaches the measurement location.

A statistical analysis has been performed on the comparison of the time response curve velocities. From this analysis, a statistically-based relationship between the velocity measured with the probe and the theoretical velocity, determined by Poisenille's solution for viscous flow, has been developed. This relationship also includes the duration of the pulse.

The investigations presented in this thesis and the development of the velocity probe by Turan have begun the next step in determining the remaining microscopic quantities needed to measure the dispersive flux term

$$
\mathbf{D}^{\star}=\left(\mathbf{v}^{\prime \alpha} c^{\prime \alpha}\right)-D_{d}\left(\nabla \bar{c}^{\alpha}+\tau\right) \text {. }
$$

\subsection{Suggestions for Future Research}

We now have a better understanding of the velocity probe proposed by Turan. However, the study performed was not exhaustive. This improved velocity probe conld be developed further and nsed to study other related phenomenon such as dispersion.

All of the experiments presented in this thesis were performed in the capillary tube system. A possible future study would be to implement a small-scale packed column experiment and test the relationship developed in this thesis. Also, there 
are still other parameters that have not been studied that might give more insight into the inner workings of the measurement method. One possibility is the effects of the change in current and the change in voltage of the pulse on the slug. This would involve increasing the number of experiments performed by a factor of 36 , if one were to follow the experimental design used in this thesis.

Another possibility is to study the actual size of the slug of fluid created by the pulse. A method for determining this conld be derived from the Poisenille flow velocity and the time from beginning to end of the breakthrough curve. Dividing the Poisenille flow velocity by the measured time wonld give a distance that wonld represent the axial width of the slug at the point of the measurement location. By performing this type of experiment at different distances downstream of the pulse wire and removing the effects of diffusion, a size of the slug at the pulse wire itself could be estimated.

As was mentioned in Section 3.4, performing the velocity experiments at different axial distance downstream of the pulse wire would also add insight to the dispersion measuring capabilities of the probe. These experiments condd be the same experiments performed to determine the size of the slug created at the pulse wire. Both of these experiments are important in determining how fast the front of the pulse propagates relative to the center of mass and if there is some enhanced propagation due to electric field effects when the de-voltage is applied to the wire.

The most important of these suggestions is the transition from the capillary tube experiments to the porous media packed column experiments. This transition is required to finish the research that was started by Nielson in 1988. 


\section{Appendix A}

\section{Programs}

\section{A.1 VEL.PRG}

ECHO.ON

Ifile: vel.prg

1 author: J. Christopher Bianchi

I date: 6/12/92

$\backslash$ Program runs experiments for a specified number of repetitions. ECHO. OFF
20 STRING FILENAME1
20 STRING FILENAME2
5 STRING FILETMP
10 STRING FILETMP2
1 STRING LETTER

$\backslash$ Define equipment on the GPIB bus

27 GPIB.DEVICE MULTIMETER \ define the multimeter as a gpib device $\backslash$ with an address of 27 .

3 GPIB.DEVICE SWITCH \ define the Fiberoptic Switch as a gpib

$\backslash$ device with an address of 3 .

11 GPIB.DEVICE VOLTAGE \ define the Voltage Source as a gpib

$\backslash$ device with an address of 11 .

17 GPIB.DEVICE SCANNER \define the Scanner as a gpib device

$\backslash$ with an address of 17 .

$\backslash$ Commands to initialize the GPIB for bus communication SEND. INTERFACE. CLEAR

REMOTE. ENABLE. ON 
: ND \Command ND to reset normal display

NORMAL.DISPLAY

;

: PREPARE.MULTIMETER \ set Multimeter at desired functions MULTIMETER

ASCII X EOS.CHARACTER

EOS.ON

"FO" GPIB. WRITE

"RO" GPIB.WRITE

"WO" GPIB. WRITE

"T4" GPIB.WRITE

"S2" GPIB.WRITE

"X" GPIB.WRITE

;

: PREPARE.VOLTAGE \ set the Voltage Source at desired functions VOLTAGE

ASCII X EOS.CHARACTER

EOS.ON

EOI.ON

"RO" GPIB.WRITE

"T4" GPIB.WRITE

" DO" GPIB.WRITE

"PO" GPIB.WRITE

" $X "$ GPIB.WRITE

;

5 STRING SCAN.DUR \variable used in PREPARE.SCANNER

Ito set pulse duration.

: PREPARE.SCANNER \ set the Scanner at desired functions SCANNER

ASCII X EOS.CHARACTER

EOS.ON

EOI.ON

"A1" GPIB.WRITE I sets the Scanner on 1-pole mode

" DO" GPIB.WRITE \ display channel number on the front panel 


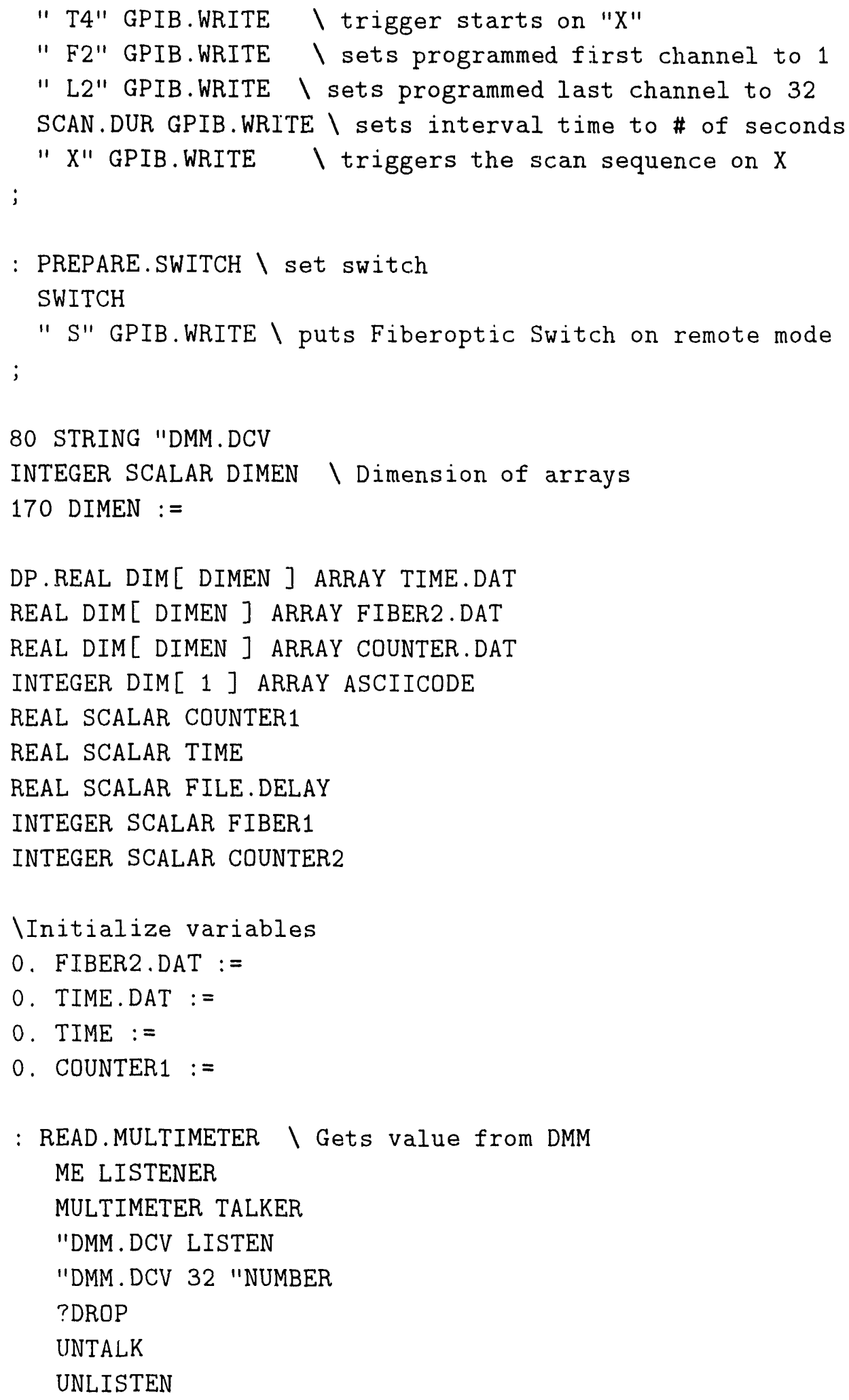




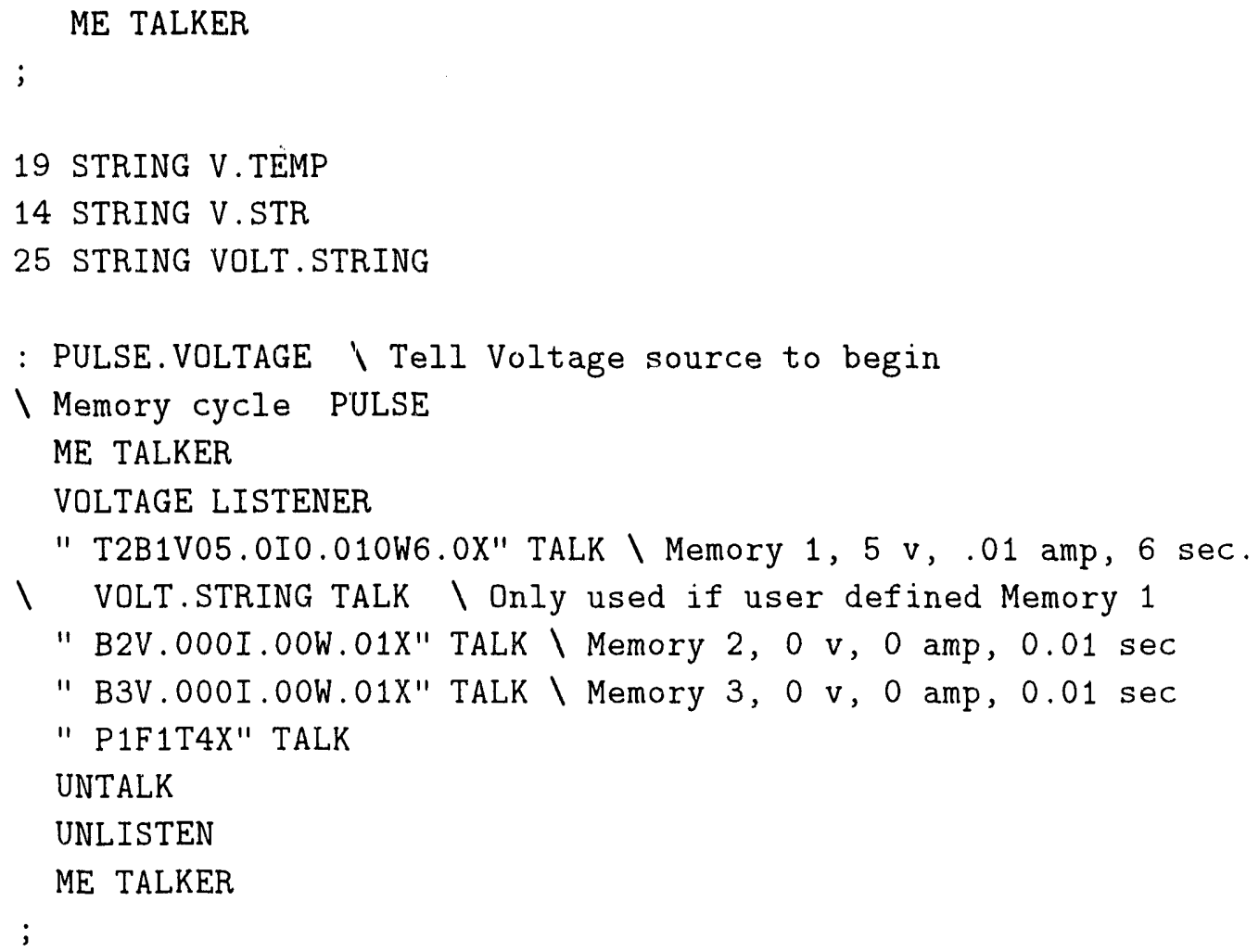




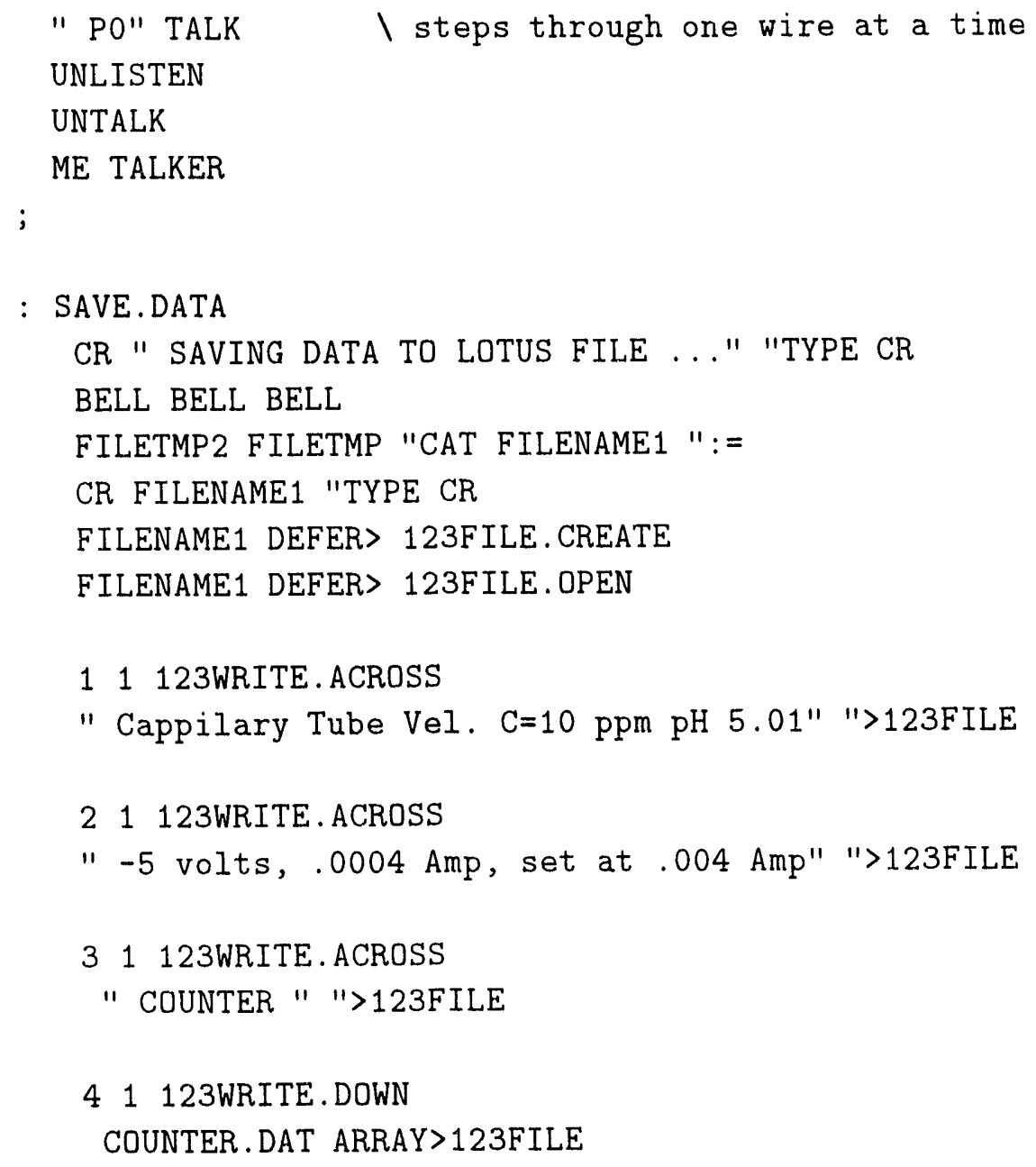


REAL SCALAR YMIN \variables for screen plot

REAL SCALAR YMAX

$\begin{array}{lllll}18 & 0 & 24 & 79 & \text { WINDOW } \text { \{BOTTOM\} }\end{array}$

: PLOT.DATA

I CR " ENTER MIN Y VALUE FOR SCALE " "TYPE

1 \#INPUT YMIN : = 0.5 YMIN $:=$

$\$ CR " ENTER MAX Y VALUE FOR SCALE " "TYPE

1 \#INPUT YMAX := 3.0 YMAX ::=

1 Graphics setup

GRAPHICS. DISPLAY

\{BOTTOM\}

$\begin{array}{lll}0.0 & 0.28 \text { VUPORT.ORIG }\end{array}$

$\begin{array}{lll}1.0 & 0.72 \text { VUPORT.SIZE }\end{array}$

SCREEN. CLEAR

VUPORT . CLEAR

3 COLOR

OUTLINE

6 FOREGROUND

$\backslash$ Set axis defaults

AXIS.DEFAULTS

$\backslash$ VERTICAL AXIS.FIT.OFF GRID.OFF

LABEL. SCALE. OFF

$\backslash$ HORIZONTAL AXIS.FIT.OFF GRID.OFF

LABEL. SCALE. OFF

$\backslash$ Position axis

NORMAL . COORDS

0.10 .12 AXIS.ORIG

$0.1 \quad 0.12$ AXIS.POINT

$0.8 \quad 0.8$ AXIS.SIZE 
i Set the number of tick mark and data ranges

255 AXIS.DIVISIONS

VERTICAL YMIN YMAX WORLD.SET

HORIZONTAL -5 120. WORLD.SET

\ Labeling the tick marks

HORIZONTAL 05 LABEL.POINTS

VERTICAL 02 LABEL.POINTS

VERTICAL -1. 05 LABEL. FORMAT

0 O TICK. JUST

$\backslash$ Plot the axes

1 COLOR

XY.AXIS . PLOT

$\backslash$ Labeling the plot

NORMAL . COORDS

.400 .960 POSITION "BREAKTHROUGH CURVES ( $Q=0.17 \mathrm{ml} / \mathrm{min})$ " LABEL

.015 .250 PUSITION 90 CHAR.DIR 90 LABEL.DIR "DC VOLT" LABEL

.470 .030 POSITION O CHAR.DIR O LABEL.DIR "TIME (sec.)" LABEL "." SYMBOL

WORLD . COORDS

;

INTEGER SCALAR REPIT

DP. REAL SCALAR LOOP.DEL:Y

DP. REAL SCALAR LOOP.DURATION

DP.REAL SCALAR LOOP.START

DP. REAL SCALAR LOOP. TIME

DP. REAL SCALAR EXP.ST.TIME

DP. REAL SCALAR EXP.RN.TIME

: cirUP \Get Experiment Run parameters for program

CR " ENTER FILE DRIVE:\FLOW_PULSE NAME FOR LOTUS ..." "TYPE CR 
"INPUT FILETMP2 ":=

1 " A:C3A1025" FILETMP "CAT FILENAME1 ":=

CR " ENTER NUMBER OF REPITITIONS AT THIS FLOW AND PULSE " "TYPE \#INPUT REPIT :=

CR " ENTER PULSE DURATION TIME FOR SCANNER (IE. W1.00) " "TYPE "INPUT SCAN.DUR ":=

CR " ENTER DELAY BETWEEN EXPERIMENT RUNS (SECONDS) " "TYPE

\#INPUT FILE.DELAY :=

FILE.DELAY $1000 *$ FILE.DELAY :=

;

\****** MAIN PROGRAM $* * * * * * * * * * * * * * * * * * * * * * * * * * * * * * * * * * * * * * *$

: VEL IVEL Command to start main program

0 COUNTER2 :=

SETUP

PREPARE. MULTIMETER

PREPARE. VOLTAGE

PREPARE. SWITCH

PREPARE. SCANNER

REPIT O DO \Repeat loop from 0 to REPIT

1 CHANNEL. NUMBER :=

FIBER. SWITCH

STACK. CLEAR

$\backslash$ Re-Initialize parameters

0 . LOOP.DELAY :=

0 . LOOP.DELAY $:=$

0 . LOOP.START $:=$

0 . LOOP.DURATION :=

0 . LOOP.TIME : =

0 . COUNTER $1:=$

PLOT.DATA

REL.TIME EXP.ST.TIME := 
STACK. CLEAR

DIMEN 0 DO \Loop from 0 to DIMEN

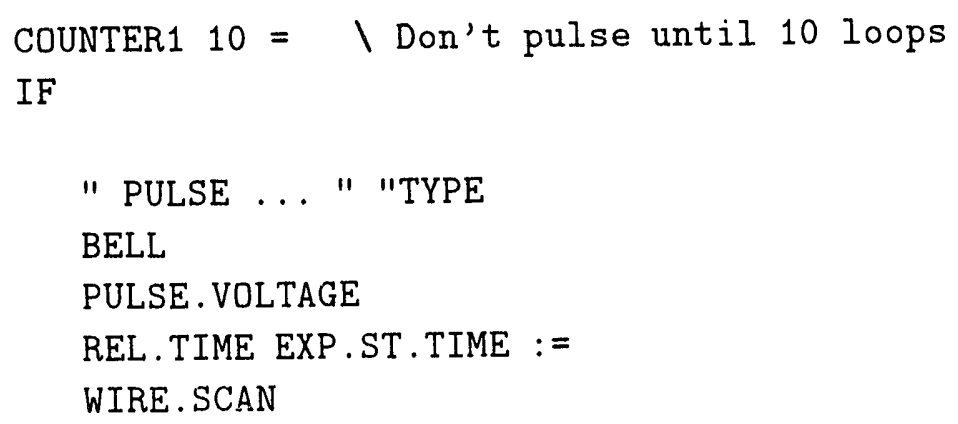




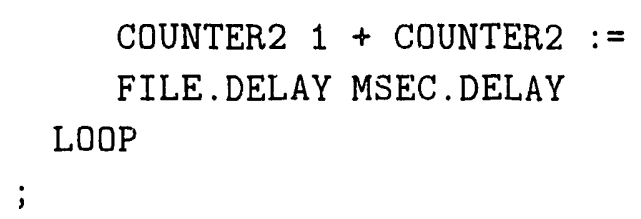

\section{A.2 NAWK Programs}

\section{A.2.1 Norm.awk}

Norm.awk is a program that normalizes the response by subtracting the average of the first nine readings from all of the readings. Essentially, it is substrating the background readings ont of the data.

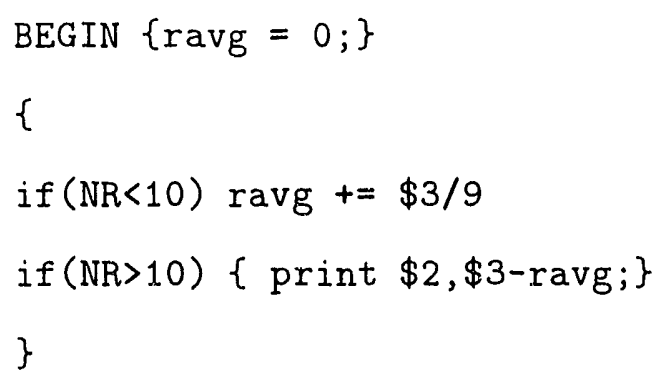

\section{A.2.2 Fstarv.awk}

This program finds the First Arrival Time by performing a moving linear regression of five consecutive points. When the slope of the linear regression is greater than $50 \%$ it defines that point as the first arrival time.

The program variables are defined below.

$t_{i}$ and $r_{i}$ are the time and response values respectively.

NR is the record number, 
$\$ 1$ and $\$ 2$ correspond to columns one and two.

sx2 is the sum five times squared.

sx is the sum of five times.

sxy is the sum of five times multiplied by their corresponding response value. sy is the sum of five response values.

slope is the slope of the line fit through five consecutive points.

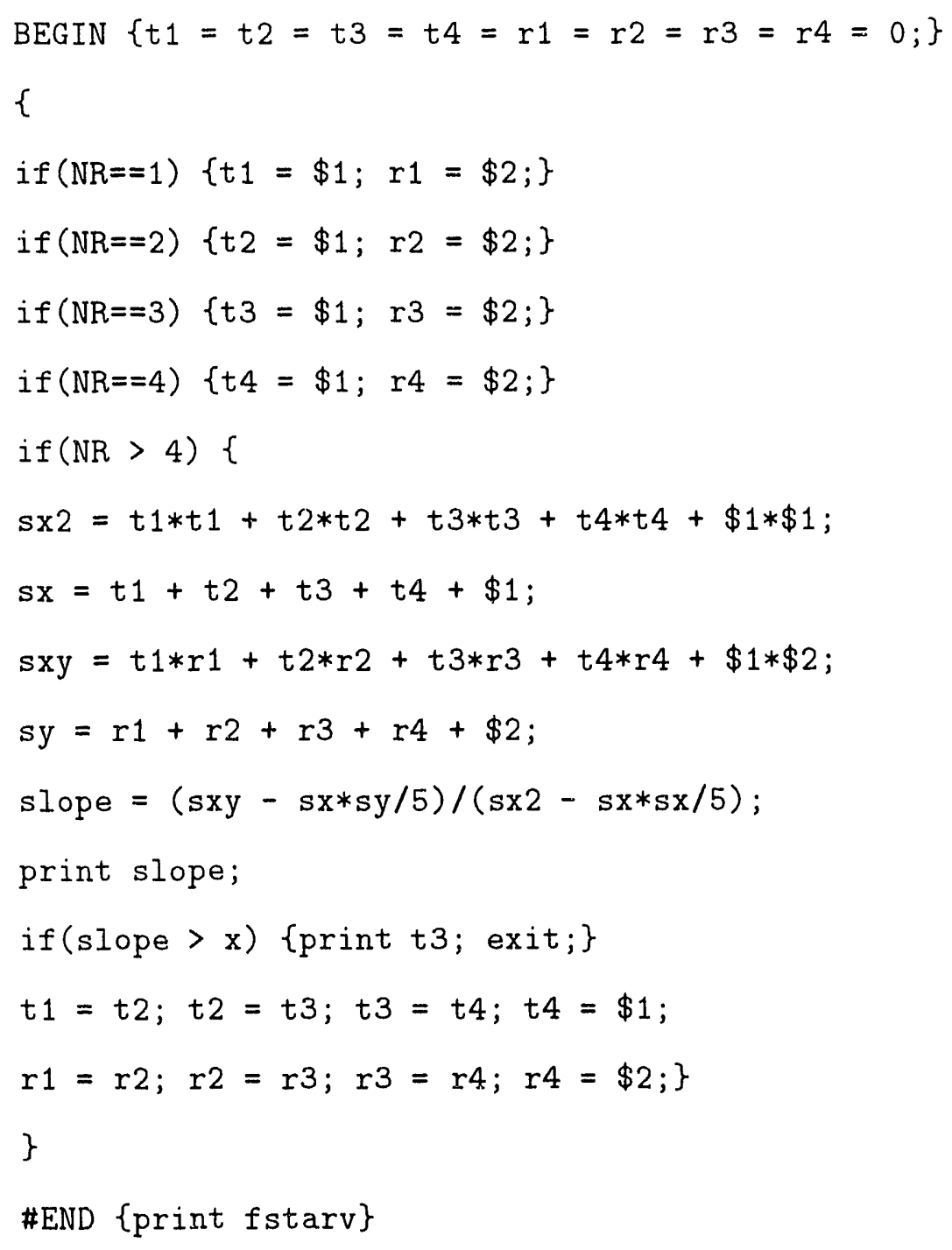




\section{Appendix B Running the Program}

This appendix outlines the steps involved in running the ASYST language, loading the velocity program (VEL.PR(x) and rumning it. The commands on numbered lines in bold type are the commands the user types and the normal type is the prompt the user will see. The $\Leftarrow$ symbol will serve as the return (or enter) key. The user is referred to the ASYST documentation for installing the software and general setup.

\section{Starting ASYST}

You must start ASYST from the ASYST directory. If you installed it in some other drive and directory other then $\mathbf{C}: \backslash \mathbf{A S Y S T}$, then you would substitute your directory for mine.

1. $\because \backslash>$ CD C: $\backslash$ ASYST $\Leftarrow$

2. $\because \backslash A S Y S T>$ ASYSTNEW $\Leftarrow$

3. Here you will be prompted to Press any key to continue.

\section{Loading and running the VEL.PRG program}

\section{OK LOAD VEL.PRG $\Leftarrow$}

You will see the title of the program and some other information, then lots of dots will appear on the screen.

5. OK VEL $\Leftarrow$

ENTER FILE IDRIVE: \FLOW_PULSE NAME FOR LOTISS ...

6. Some file name $\Leftarrow$

Only use seven characters for the file name because the program puts on the last character as an $\mathrm{A}$ for the first repetition and, for example, an $\mathrm{E}$ for the fifth repetition.

ENTER NUMBER OF REPETITIONS AT THIS FLOW AND PILSE

7. $5 \Leftarrow$

ENTER PULSE DURATION TIME FOR SCANNER (IE. W1.00) 
$8 . \mathbf{1 . 5 0} \Leftarrow \quad$ (for 1.5 seconds)

ENTER DELAY BETWEEN EXPERIMENTS RUNS (SECONDS')

$9.60 \Leftarrow \quad$ (for one minute between each repetition)

For this example, the program will run five repetitions with a 1.5 second pulse duation and a one minute delay between each repetition. 


\section{References}

[Acr89] Michael Acrivlellis. Determination of the magnitudes and signs of flow parameters by hot-wire anemometry. Part I. measurements using hot-wire x probes. Review of S'cientific Instruments, 60(7):1, 1989.

[Bea79] Jacob Bear. Hydraulics of Croundwater- (Water resources and cnvironmental engineering). Mc(iraw-Hill Inc, 1979.

[BNF90] H. H. Brumu, N. Nabhani, and A. A. Fardad. Velocity component measurements by $\mathrm{x}$ hot-wire anemometry. Measurement Science and Technology, 1(12):1314, 1990.

[BP85] M.S. Beck and A. Plaskowski. Cross-Correlation Flowmeters - The ir Design and Application. Bristol: Adam Hilger, 1985.

[Dur77] T. S. Durrani. Laser Systems in Flow Measurement. Plenum Press, 1977.

[Grill] Albert Griffiths. On the movement of a coloured index along a capillary tube, and its application to the measurement of the circulation of water in a closed circuit. Procedings of the Physical Society of London, 23:190-197, 1911.

[HDM84] T. Hirschfeld, T. Deaton, and F. Milanovich. The Feasibility of Using Fiber Optics For Monitoring Croundwater Contaminants. Technical report, Environmental Monitoring Systems Laboratory, January 1984. EPA Resoarch and Development Report.

[H(779] Majid Hassanizadeh and William G. Gray. General conservation equations of multi-phase systems: 1. averaging procedure. Advances in Water Resources, September 1979.

[JS91] Kyung-Young Jhang and Takuso Sato. 3-d velocity field measurement using multiple ultrasonic plane detections and high-order correlation analysis. IEEE Transactions on Ultrasonics, Ferroclectrics, and Frequency Control, 38(2):93-99, 1991.

[KBA87] T. J. Kulp, D. Bishop, and S. M. Angel. Column profile measurements using fiber-optic spectroscopy. July 1987. preprint. 
[LP86] Lawrence Livermore National Laboratory and Princeton Iniversity. Porous media species tramsport at the microscopic and macroscopic scales. A proposal for research to the U.S. Department of Energy, October 19, 1986.

[LPS75] D. A. Lane, I.(\%. Patel, and .J. A. Sirs. The variation of indicator dilution curves with velocity profile. Physics in Medicine and Biology, $20(4):(613-623,1975$.

[MT79] W. P. Mason and R. N. Thurston. Physical Acoustics XIV. New York Academic, 1979.

[MW82] W. H. Munk and ( $:$ Wunsch. Up/down resolution in aconstic tomography. Deep-Sea Res, 29(12A):1415-1436, 1982.

[Nie88] Jon Mark Nielsen. A Study of the Spatial Variability of Microscopic Solute Transport in Dispersive Flows Using Fiber-Optic Sensors. Master's thesis, Princeton University, June 1988.

[NPLA91] Jon Mark Nielsen, George F. Pinder, Thomas J. Kulp, and Stanley M. Angel. Investigation of dispersion in porous media using fiber-otic technology. Water Resoures Research, 27(10):2743-2749, October 1991 .

[Pin91] Cieorge F. Pinder. Hydrophysical Processes in the Subsurface: Investigations of Organic (hemical Transport Using New Scnsor Technologies. A proposal requesting renewal for project, February $7,1991$.

[Ste94] (i. N. Stewart. Researches on the circulation time in organs and on the influences which alfect it. The Journal of Physiology, 15:1-89, 1894.

[SW 85] Victor L. Streeter and E. Benjamin Wylie. Fluid Mechanics. McCraw-Hill Book Company, 1985.

[Tay53] Sir Geoffrey Taylor. Dispersion of soluble matter in solvent flowing slowly through a tube. Proc. of the Royal Society of London, Series A, 219:186-203, Angust 195:3.

[TC 186] Andrew F.B. Tompson and William G. Gray. A second-order approach for the modeling of dispersive transport in porous media

1. theoretical development

2. application to solute motion in pipes and capillary tubes. Water Resources Research, 22(5):591-599, 601-614, May 1986. 
[VWB91] Petar Vukoslavervic, James M. Wallace, and Jean-Lonis Balint. The velocity and vorticity vector fields of a turbulent boundary layer. part 1. simulaneons measurement by hot-wire anemometry. Journal of Fluid Mechanics, 228:25, 1991.

[Whi47] H. L. White. Measurement of cardiac output by a continuously recording conductivity method. The American Journal of Physiol$o g y, 151: 45-57,1947$. 

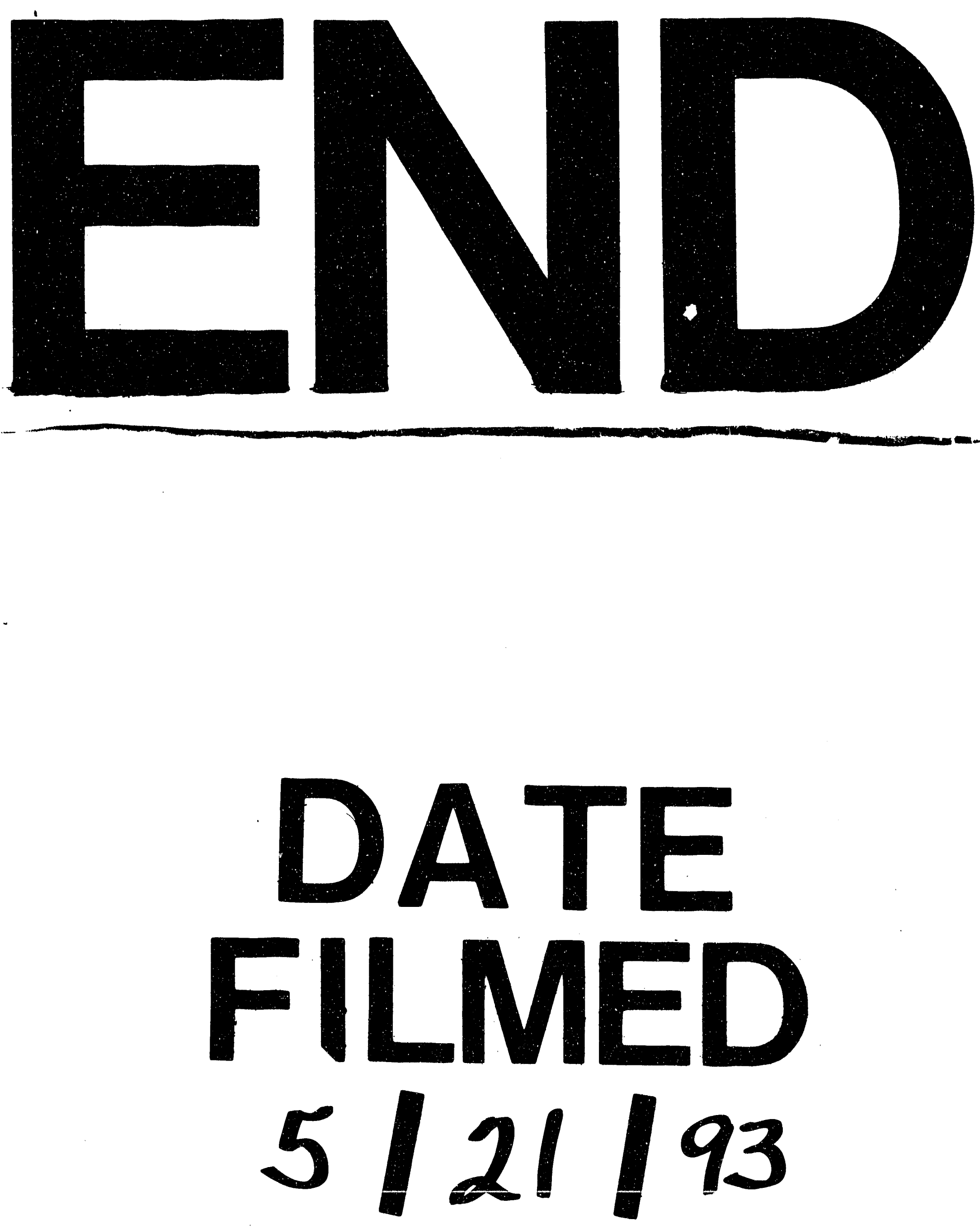
